\title{
Radial Distribution of X-ray Point Sources Near the Galactic Center
}

\section{Citation}

Hong, JaeSub, Maureen van den Berg, Jonathan E. Grindlay, and Silas Laycock. 2009. "RADIAL DISTRIBUTION OF X-RAY POINT SOURCES NEAR THE GALACTIC CENTER." The Astrophysical Journal 706 (1): 223-37. https://doi.org/10.1088/0004-637x/706/1/223.

\section{Permanent link}

http://nrs.harvard.edu/urn-3:HUL.InstRepos:41399744

\section{Terms of Use}

This article was downloaded from Harvard University's DASH repository, and is made available under the terms and conditions applicable to Other Posted Material, as set forth at http:// nrs.harvard.edu/urn-3:HUL.InstRepos:dash.current.terms-of-use\#LAA

\section{Share Your Story}

The Harvard community has made this article openly available.

Please share how this access benefits you. Submit a story.

Accessibility 


\title{
RADIAL DISTRIBUTION OF X-RAY POINT SOURCES NEAR THE GALACTIC CENTER
}

\author{
JaeSub Hong $^{1}$, Maureen van den Berg ${ }^{1}$, Jonathan E. Grindlay ${ }^{1}$, and Silas Laycock ${ }^{2}$ \\ ${ }^{1}$ Harvard-Smithsonian Center for Astrophysics, 60 Garden St., Cambridge, MA 02138, USA; jaesub@ head.cfa.harvard.edu \\ ${ }^{2}$ Gemini Observatory, 670 N. A'ohoku Place, Hilo, HI 96720, USA \\ Received 2009 February 18; accepted 2009 August 31; published 2009 October 29
}

\begin{abstract}
We present the $\log N-\log S$ and spatial distributions of X-ray point sources in seven Galactic bulge (GB) fields within $4^{\circ}$ from the Galactic center (GC). We compare the properties of 1159 X-ray point sources discovered in our deep (100 ks) Chandra observations of three low extinction Window fields near the GC with the X-ray sources in the other GB fields centered around Sgr B2, Sgr C, the Arches Cluster, and Sgr A* using Chandra archival data. To reduce the systematic errors induced by the uncertain X-ray spectra of the sources coupled with field-and-distancedependent extinction, we classify the X-ray sources using quantile analysis and estimate their fluxes accordingly. The result indicates that the GB X-ray population is highly concentrated at the center, more heavily than the stellar distribution models. It extends out to more than 1.4 from the GC, and the projected density follows an empirical radial relation inversely proportional to the offset from the GC. We also compare the total X-ray and infrared surface brightness using the Chandra and Spitzer observations of the regions. The radial distribution of the total infrared surface brightness from the 3.6 band $\mu \mathrm{m}$ images appears to resemble the radial distribution of the X-ray point sources better than that predicted by the stellar distribution models. Assuming a simple power-law model for the $\mathrm{X}$-ray spectra, the closer to the GC the intrinsically harder the X-ray spectra appear, but adding an iron emission line at $6.7 \mathrm{keV}$ in the model allows the spectra of the GB X-ray sources to be largely consistent across the region. This implies that the majority of these GB X-ray sources can be of the same or similar type. Their X-ray luminosity and spectral properties support the idea that the most likely candidate is magnetic cataclysmic variables (CVs), primarily intermediate polars (IPs). Their observed number density is also consistent with the majority being IPs, provided the relative $\mathrm{CV}$ to star density in the GB is not smaller than the value in the local solar neighborhood.
\end{abstract}

Key words: Galaxy: bulge - Galaxy: center - stars: statistics - X-rays: binaries - X-rays: stars

Online-only material: color figures, machine-readable table

\section{INTRODUCTION}

The Chandra X-ray Observatory has opened a new era in studies of the X-ray source population in the Galactic bulge (GB). A series of shallow and deep Chandra observations in the Galactic center $(\mathrm{GC})$ region have revealed $\sim 1000 \mathrm{X}$-ray point sources in a $2^{\circ} \times 0.8$ region (Wang et al. 2002) and $2357 \mathrm{X}$-ray point sources in a $17^{\prime} \times 17^{\prime}$ region around the Sgr A* (Muno et al. 2003, hereafter M03). An additional $\sim 2000$ sources found in the Bulge Latitude Survey (BLS; two $0.8 \times 1.5$ regions) provide the initial results for the latitude distribution of the GB sources (J. E. Grindlay et al. 2009, in preparation). The $\mathrm{X}$-ray luminosities and relatively hard spectra ruled out that the majority of the GC X-ray point sources are normal stars, active binaries, young stellar objects, or quiescent low-mass $\mathrm{X}$-ray binaries (qLMXBs; M03). From the lack of real matches between the bright infrared (IR; $K<15$ ) and X-ray sources in the Sgr A* field, Laycock et al. (2005, hereafter L05) concluded that high-mass X-ray binaries (HMXBs) cannot account for more than $10 \%$ of the X-ray sources in this region. While the leading candidate that fits the properties of these $\mathrm{X}$-ray sources is now magnetic cataclysmic variables (CVs; Muno et al. 2004, L05), the relatively hard X-ray spectra of some of the most recently discovered qLMXBs imply qLMXBs could be misrecognized as CVs and be more common in the GB than thought in the past (Wijnands et al. 2005; Bogdanov et al. 2005). Infrared searches for the counterparts of these GB X-ray sources have been actively pursued (e.g., Muno et al. 2005), but the exact nature of the majority of the sources is still elusive due to high obscuration by dust and source confusion by the high star density.
We have conducted a series of deep (100 ks) Chandra observations of three low extinction Window fields-Baade's Window (BW), Stanek's Window (SW; Stanek 1998), and the "Limiting Window" (LW) - near the GC (Section 2). These Window fields allow us to observe the GB X-ray population and their Galactic radial distribution with minimal obscuration by dust. We have discovered $1159 \mathrm{X}$-ray point sources in these fields. We compare their distributions with $\mathrm{X}$-ray sources in other GB fields-the Sgr B2, Sgr C, Arches Cluster, and Sgr $A^{*}$ fields. We present a new approach using quantile analysis (Section 3) to minimize the systematic errors in flux estimation, to classify sources by their X-ray spectral types and investigate their radial distribution. We compare the $\mathrm{X}$-ray distribution with the known models of the stellar distribution (Section 4) and investigate the nature of the X-ray population (Section 5); see also van den Berg et al. (2009), where we put constraints on the nature of the X-ray source populations from the optical point of view, using Hubble Space Telescope (HST) observations of the Window fields taken simultaneously with the Chandra exposures. This work is part of our Chandra Multi-wavelength Plane (ChaMPlane) Survey designed to measure the space density and probable nature of the low-luminosity accretionpowered sources in the Galaxy (Grindlay et al. 2005).

\section{OBSERVATIONS AND DATA ANALYSIS}

We performed Chandra/ACIS-I observations of the BW on 2003 July 9 (Obs. ID 3780), the SW on 2004 February 14/15 (Obs. ID 4547 and 5303), and the LW on 2005 August 19/22 and October 25 (Obs. ID 5934, 6362 and 6365). Due to technical 
Table 1

X-ray Point Sources in the Selected GB Fields

\begin{tabular}{|c|c|c|c|c|c|c|c|c|c|c|}
\hline \multirow[t]{2}{*}{ Field } & \multirow[t]{2}{*}{ Obs. ID } & \multirow{2}{*}{$\begin{array}{c}l \\
(\operatorname{deg})\end{array}$} & \multirow{2}{*}{$\begin{array}{c}b \\
(\operatorname{deg})\end{array}$} & \multirow{2}{*}{$\begin{array}{l}\text { Offset }^{\mathrm{a}} \\
(\mathrm{deg})\end{array}$} & \multirow[t]{2}{*}{$N_{\mathrm{H} 22}{ }^{\mathrm{b}}$} & \multirow{2}{*}{$\begin{array}{l}\mathrm{GTI}^{\mathrm{c}} \\
(\mathrm{ks}) \\
\end{array}$} & \multicolumn{4}{|c|}{ Source Count ${ }^{\mathrm{d}}$} \\
\hline & & & & & & & $B_{X}$ & $S_{X}$ & $H_{X}$ & Combined \\
\hline BW & 3780 & 1.06 & -3.83 & 3.93 & 0.31 & 96 & 365 & 326 & 134 & 407 \\
\hline SW & 4547,5303 & 0.25 & -2.15 & 2.12 & 0.48 & 96 & 388 & 313 & 140 & 433 \\
\hline LW & $5934,6362,6365$ & 0.10 & -1.43 & 1.39 & 0.68 & 94 & 282 & 184 & 174 & 319 \\
\hline Sgr B2 & 944 & 0.59 & -0.03 & 0.65 & 81.2 & 97 & 279 & 126 & 224 & 363 \\
\hline Sgr C & 5892 & -0.57 & -0.02 & 0.51 & 52.7 & 97 & 313 & 188 & 241 & 442 \\
\hline Arches & 4500 & 0.12 & -0.02 & 0.19 & 52.5 & 97 & 330 & 84 & 328 & 423 \\
\hline $\begin{array}{l}\text { Sgr A* } \\
\text { (Stackede) }\end{array}$ & 3665 & -0.06 & -0.05 & - & 56.5 & $\begin{array}{c}88 \\
698\end{array}$ & $\begin{array}{c}401 \\
2251\end{array}$ & $\begin{array}{c}92 \\
370\end{array}$ & $\begin{array}{c}400 \\
2316\end{array}$ & $\begin{array}{c}508 \\
2876\end{array}$ \\
\hline
\end{tabular}

Notes. ${ }^{a}$ The aim point offset from $\mathrm{Sgr} \mathrm{A}^{*}$.

b The estimates for the integrated neutral hydrogen column density along the line of the sight (in $10^{22} \mathrm{~cm}^{-2}$ ) by Schlegel et al. (1998) for the location of the aim point. This is only for guiding purpose due to the large uncertainty in the Galactic plane fields.

${ }^{c}$ The good time intervals (GTIs). The total exposure (i.e., before cleaning) is $100 \mathrm{ks}$ each (750 ks for the stacked Sgr A* field).

$\mathrm{d}$ The number of the sources with net count $\geqslant 1$ in the broad band $(0.3-8 \mathrm{keV})$ on the ACIS-I CCDs $(0,1,2$, and 3$)$ in the three detection bands $\left(B_{X}: 0.3-8 \mathrm{keV}, S_{X}: 0.3-2.5 \mathrm{keV}, H_{X}: 2.5-8 \mathrm{keV}\right)$ and the combined unique source list.

e Fourteen pointings are stacked, and they are Obs. ID 242, 2951, 2952, 2953, 2954, 2943, 3663, 3392, 3393, 3549, 3665, 4683, 4684, and 5360 .

constraints, the SW and LW observations were segmented into a few pointings, which we stacked for further analysis. Table 1 summarizes the observational parameters and X-ray source statistics of the Window and other GB fields analyzed in this paper. For the $\mathrm{Sgr} \mathrm{A}^{*}$ field, we use the results from a $100 \mathrm{ks}$ observation (Obs. ID 3665) for easy comparison with other GB fields that were observed with similar exposure times, and we have also stacked 14 observations from the archive, totaling $750 \mathrm{ks}$ exposure.

We have analyzed the data as a part of the ChaMPlane survey. For uniform analysis of all the ChaMPlane fields, we have developed a series of X-ray processing tools, mainly based on version 3.4 of the CIAO package (Hong et al. 2005, hereafter H05). ${ }^{3}$ After initial screening of the CXC level-2 data (e.g., select the events in good time intervals during which the background fluctuates $<3 \sigma$ above the mean level), we detect $\mathrm{X}$-ray point sources with a wavelet algorithm (wavdetect; Freeman et al. 2002) with a significance threshold of $10^{-6}$. The wavdetect routine is run on each individual observation and the stacked data set if available. Multiple observations are considered stackable (the SW, LW, and Sgr A* fields here) if the aim points are on the same detector (ACIS-I or ACIS-S) and they are within $1^{\prime}$ of each other.

In $\mathrm{H} 05$, we used source detections in the broad $\left(B_{X}: 0.3-\right.$ $8.0 \mathrm{keV})$ band. We now also incorporate source detections in the soft $\left(S_{X}: 0.3-2.5 \mathrm{keV}\right)$ and hard $\left(H_{X}: 2.5-8.0 \mathrm{keV}\right)$ bands in addition to the broad band. We establish a unique source list by cross matching the three detection lists based on the relative distance of possibly identical source pairs (the closest pairs) in the three different bands. The relative distance $\left(d_{r}\right)$ of two sources is defined by the ratio of the source distance to the quadratic sum of the positional errors. Note that there is no astrometric offset among the images in the three bands. The positional errors of sources are calculated by an empirical formula based on the $\mathrm{MARX}^{4}$ simulations (Equation (5) in H05).

Establishing a unique source list is straightforward in relatively un-crowded fields such as the Window fields, but it can

\footnotetext{
3 Some of the fields were processed by the tools based on version 3.1 of the CIAO package, but the difference between two versions is minimal.

4 http://space.mit.edu/CXC/MARX/
}

be tricky in heavily populated fields and in very deep exposures such as the stacked Sgr A* data set. Figure 1 shows the distributions of the relative separations of nearest-neighbor source pairs among the three detection bands, $S_{X}, H_{X}$, and $B_{X}$. As examples, we compare the $100 \mathrm{ks}$ observations of the Stanek Window and Sgr A* field, and the $750 \mathrm{ks}$ stacked data set of the Sgr A* field. A source detection in each band contributes two pairs to the distribution, one from each of the other two bands.

The bimodal shape of the distribution indicates two types of the pairs contribute to the distribution: one type consists of the truly identical sources detected in different bands and the other consists of random pairs of unrelated sources. The distribution of the random pairs can be estimated by introducing an arbitrary astrometric offset in the source position between the detection bands. The (blue) dashed-dotted line in Figure 1 shows such an example ( $1^{\prime}$ offset in both right ascension (R.A.) and declination (decl.)), the shape of which closely resembles the right side of the bimodal distribution of original sources. The slight excess over the original distribution is due to the real pairs being transformed into new random pairs by the positional offset.

After visual inspection of the raw images and the distributions of the relative distances in Figure 1, we use a simple cut $\left(d_{r} \leqslant 2.0\right.$; red vertical line) for establishing the unique source list. The cut is sufficient for identifying virtually $100 \%$ of the unique sources detected in multiple bands. From the distributions of the relative distances of the random pairs, we estimate that the false random matches surviving the cut ranges from 10 to 25 for the $100 \mathrm{ks}$ observations. The corresponding number of independent sources that might have lost by the false random matches ranges from 5 to $10(\sim 1 \%)$ for the $100 \mathrm{ks}$ observations and about 100 for the stacked Sgr A* field ( 3\%).

When multiple detections in the three bands are identified as a unique source, we select the one with the smallest positional error for the unique source list. We note that the final source position and error could be derived from a form of weighted average of astrometric properties of multiple detections. However, the $S_{X}$ and $H_{X}$ band detections are not entirely independent from the $B_{X}$ band detection. Therefore, in order to avoid unnecessary complication in the analysis, we simply take the astrometric (and photometric) properties of the source with the smallest 

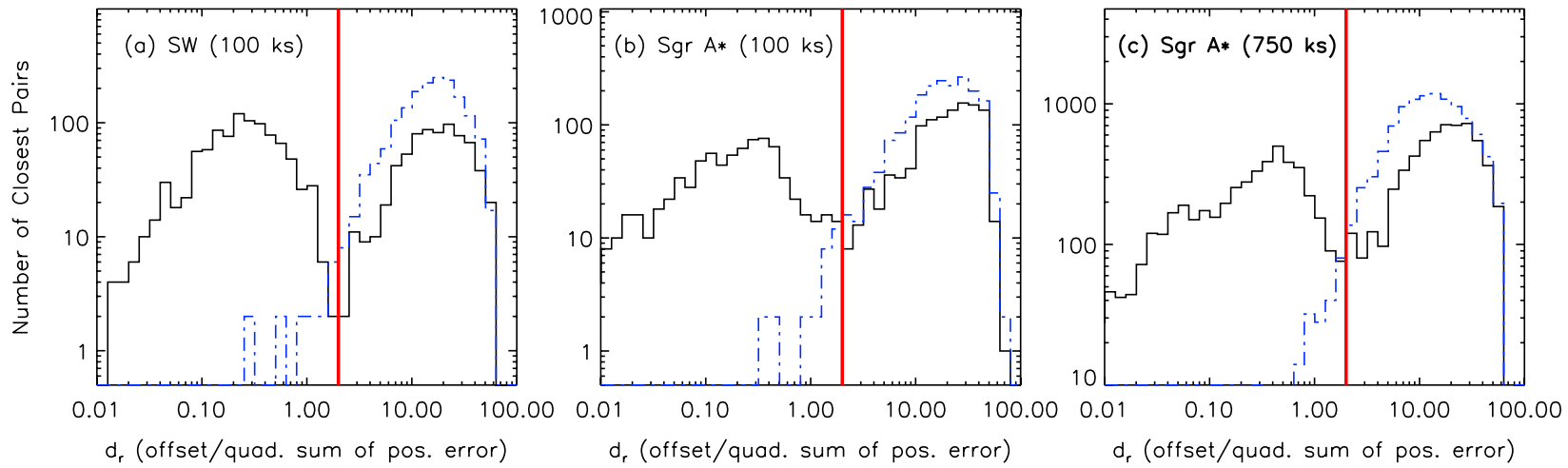

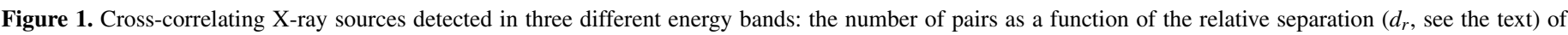

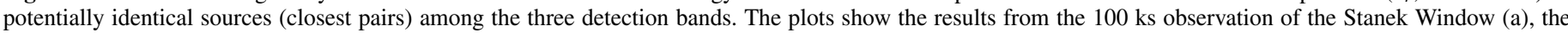

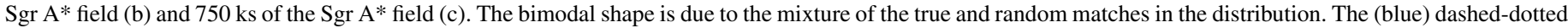

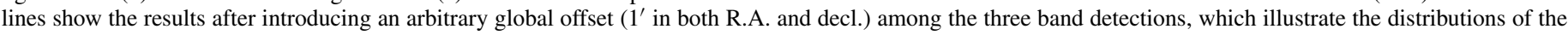
random matches.

(A color version of this figure is available in the online journal.)

positional errors, which is the detection with the highest significance among the three bands.

As a sanity check, we compare our detection with the available catalogues in the literature. M03 provided a catalog of the 2357 $\mathrm{X}$-ray point sources discovered in the $690 \mathrm{ks}$ exposure ( $626 \mathrm{ks}$ of GTIs) of the Sgr A* field, Muno et al. (2006, hereafter M06) for the 397 point sources in the $100 \mathrm{ks}$ exposure of the Sgr B2 field (Obs. ID 944) and Wang et al. (2006, hereafter W06) for the 244 $\mathrm{X}$-ray point sources in the $100 \mathrm{ks}$ exposure of the Arches Cluster (Obs. ID 4500). The majority ( $25 \%-90 \%)$ of these sources are also detected in our analysis or vice versa if our source list is shorter than theirs. A small fraction of the sources are missing due to many subtle differences in the detection methods and the selection criteria for the lists such as the detection energy bands $(0.5-8,0.5-1.5$, and $4-8 \mathrm{keV}$ in M03 or 1-4, 4-9, and 1-9 keV in W06), the pointing or GTI selections (e.g., Obs. ID 1561 was not included in our analysis in the stacked Sgr A* field). In addition, in the case of W06, their high significance threshold $\left(10^{-7}\right.$ except for the inner $2^{\prime} \times 2^{\prime}$ region, about 10 counts in their broad band, $1-9 \mathrm{keV}$ ) is one of the main reasons for the difference (244 versus 423 ) in the total number of the source detections. M03 and M06 list all the source detections including the ones with negative counts in their broad band $(0.5-8 \mathrm{keV})$. Our source list includes the detections with net counts $\geqslant 1$ in the $B_{X}$ band $(0.3-8 \mathrm{keV})$.

After source detection, we perform aperture photometry on each source to extract the basic source properties such as net count and net count rate in the conventional energy bands $\left(S_{C}: 0.5-2.0, H_{C}: 2.0-8.0\right.$, and $\left.B_{C}: 0.5-8.0 \mathrm{keV}\right)$ and energy quantiles in the broad band $\left(B_{X}: 0.3-8.0 \mathrm{keV}\right)$. For the sources that fall near other sources, we carefully revise the aperture of the source regions by excluding overlapping sections to minimize the contamination from the neighbors (H05). Table 2 lists a part of the source catalog with selected source properties used in this paper. The complete list for the Window and other GB fields is available in the electronic edition.

\section{FLUX ESTIMATION BY QUANTILE CLASSIFICATION}

In order to compare source distribution in various regions of the sky with diverse extinctions, it is necessary to correct for the interstellar absorption and use the unabsorbed source flux of individual sources. However, such a calculation is not trivial for X-ray sources with diverse spectral types as found in Galactic plane fields since faint sources are unsuitable for spectral fitting. Moreover, the relatively large extinction in the GB fields and its usually unknown field-and-distance-dependent variation make it difficult to identify the underlying $\mathrm{X}$-ray spectral model (e.g., power law versus thermal bremsstrahlung, etc.). An inaccurate assumption of the spectral model when estimating flux introduces systematic errors that often exceed the statistical errors. We therefore employ quantile analysis (Hong et al. 2004, hereafter H04), which is relatively free of the countdependent bias inherent in X-ray hardness ratio or X-ray color analysis, and so provides a better measure for classifying $\mathrm{X}$-ray sources in the GB fields. In the following, the energy quantile $E_{x}$ corresponds to the energy below which $x \%$ of the counts are detected, e.g., $E_{50}$ is the median energy.

\subsection{Quantile Analysis}

Figure 2 shows the quantile diagrams of the $\mathrm{X}$-ray sources (signal-to-noise ratio $\mathrm{S} / \mathrm{N} \geqslant 3$ in $B_{X}$ ) in the selected GB fields overlaid with grids for a simple power-law model (PL, solid lines) and a power law plus an iron emission line (PL+Fe, dashed) at $6.7 \mathrm{keV}$ with $0.4 \mathrm{keV}$ equivalent width (EW; see Section 3.2 for the motivation of the line choice). The lower right panel also includes a grid for the thermal bremsstrahlung model (TB, dotted). The $\mathrm{S} / \mathrm{N}$ here is calculated based on the statistical errors $\left(\sigma_{c}\right)$ using small-number statistics from Gehrels et al. (1986) (see also Kim et al. 2004). The difference in the model grids between PL and PL+Fe is only evident in the highly absorbed or spectrally very hard section of the diagram (the right side) because a small iron line ( $>6 \mathrm{keV}$ with $<1 \mathrm{keV} \mathrm{EW}$ ) does not make a noticeable difference in three quantiles of the soft sources.

Relatively insensitive to the extinction, the sources around $(x, y)=(-0.9,1.6)$ are present in every field and they appear unabsorbed and intrinsically soft regardless of the assumed model class (PL, PL+Fe, or TB). Foreground thermal sources such as coronally active stars fit the description. The location of relatively hard sources in the diagram varies with the field extinction. In the Window fields, the hard sources are relatively unabsorbed, but on approach to the GC, there is an increasing trend in the source number with both the average absorption and the intrinsic hardness, when compared to a simple PL model. For instance, in the BW most sources have PL photon index $(\Gamma)>1$ and $N_{\mathrm{H} 22}<1$, whereas in the LW many sources lie in $\Gamma<1$ and $N_{\mathrm{H} 22}>1$. In the Sgr $\mathrm{A}^{*}$ field and the rest, most of the hard sources are heavily absorbed with $\Gamma \lesssim 1$ and $N_{\mathrm{H} 22} \gtrsim 1$ 
Table 2

Catalog of X-ray Point Sources in the Window and Four GB Fields

\begin{tabular}{|c|c|c|c|c|c|c|c|c|c|c|c|c|c|}
\hline \multirow{2}{*}{$\begin{array}{c}\text { Source } \\
\text { Name } \\
(\text { CXOPS J) }\end{array}$} & \multirow[b]{2}{*}{ Field } & \multirow[b]{2}{*}{$\begin{array}{l}\text { R.A. } \\
(\text { deg) }\end{array}$} & \multicolumn{3}{|c|}{ Posi. } & \multicolumn{3}{|c|}{ Net Counts ${ }^{\mathrm{c}}$} & \multirow{2}{*}{$\begin{array}{c}\mathrm{S} / \mathrm{N}^{\mathrm{d}} \\
H_{C}\end{array}$} & \multicolumn{3}{|c|}{ Quantiles } & \multirow{2}{*}{$\begin{array}{c}\text { Unabsorbed Flux } \\
H_{C} \\
\left(10^{-14} \mathrm{erg} \mathrm{cm}^{-2} \mathrm{~s}^{-1}\right)\end{array}$} \\
\hline & & & $\begin{array}{l}\text { Decl. } \\
\text { (deg) }\end{array}$ & $\begin{array}{l}\text { Error }^{\mathrm{a}} \\
(\operatorname{arcsec})\end{array}$ & $\begin{array}{c}\text { Offset }^{\mathrm{b}} \\
(\operatorname{arcmin})\end{array}$ & $B_{X}$ & $S_{C}$ & $H_{C}$ & & $\begin{array}{c}E_{50} \\
(\mathrm{keV})\end{array}$ & $\begin{array}{l}\text { Quartile } \\
\text { Ratio }^{\text {e }}\end{array}$ & $\overline{\text { Group }}$ & \\
\hline $180230.4-295647$ & BW & 270.626934 & -29.946497 & 1.29 & 10.05 & $123.6(14.3)$ & $71.5(10.9)$ & $53.3(9.9)$ & 5.4 & $1.89(0.10)$ & $1.30(0.17)$ & 1 & $1.31(0.24)$ \\
\hline $180235.9-295323$ & $\mathrm{BW}$ & 270.649946 & -29.889846 & 3.32 & 9.87 & $23.6(9.6)$ & $23.4(8.1)$ & $-0.2(5.6)$ & 0.0 & $1.05(0.15)$ & $2.08(0.48)$ & 1 & $-0.01(0.13)$ \\
\hline \multicolumn{14}{|c|}{ 270 } \\
\hline 175404.4-294359 & SW & 268.518385 & -29.733089 & 2.56 & 9.58 & 34.1 (11.2) & $25.3(9.2)$ & $5.9(6.6)$ & 0.9 & $1.34(0.18)$ & $1.63(0.64)$ & 1 & $0.13(0.14)$ \\
\hline $175405.3-294717$ & SW & 268.522117 & -29.788307 & 2.47 & 8.04 & $22.2(8.9)$ & $19.7(7.7)$ & $2.9(5.1)$ & 0.6 & $1.40(0.24)$ & $1.61(0.40)$ & 1 & $0.06(0.11)$ \\
\hline \multicolumn{14}{|c|}{ (1) } \\
\hline $175051.2-293418$ & LW & 267.713518 & -29.571797 & 1.02 & 8.10 & $113.8(13.8)$ & $36.2(9.1)$ & $75.8(10.9)$ & 7.0 & $2.63(0.20)$ & $1.25(0.16)$ & 2 & $2.21(0.32)$ \\
\hline $175052.0-293319$ & LW & 267.716827 & -29.555400 & 2.92 & 8.14 & $16.4(9.2)$ & $5.2(6.8)$ & $9.3(6.6)$ & 1.4 & $0.97(5.31)$ & $0.20(0.29)$ & 2 & $0.27(0.19)$ \\
\hline $175053.3-293207$ & LW & 267.722097 & -29.535548 & 2.12 & 8.29 & $25.0(10.3)$ & $2.8(7.3)$ & $22.4(7.8)$ & 2.9 & $3.46(0.38)$ & $1.74(0.48)$ & 3 & $0.77(0.27)$ \\
\hline
\end{tabular}

Notes. This table shows a part of the complete list, which is available in the electronic edition.

a The $95 \%$ positional error radius.

b The offset from the aim point.

c The net counts based on the aperture photometry (Hong et al. 2005).

${ }^{d}$ The $\mathrm{S} / \mathrm{N}$ ratio in the $H_{C}$ band. The sources with $\mathrm{S} / \mathrm{N} \geqslant 3$ are included in the $\log N-\log S$ plot in Figure 5 .

e $3\left(E_{25}-0.3 \mathrm{keV}\right) /\left(E_{75}-0.3 \mathrm{keV}\right)$.

${ }^{\mathrm{f}}$ Based on the PL+FE model using quantile analysis. We do not include the flux estimates in the other bands due to their large uncertainty; see the text for the details. The uncertainties for net counts and fluxes are statistical errors.

(This table is available in its entirety in a machine-readable form in the online journal. A portion is shown here for guidance regarding its form and content.) 


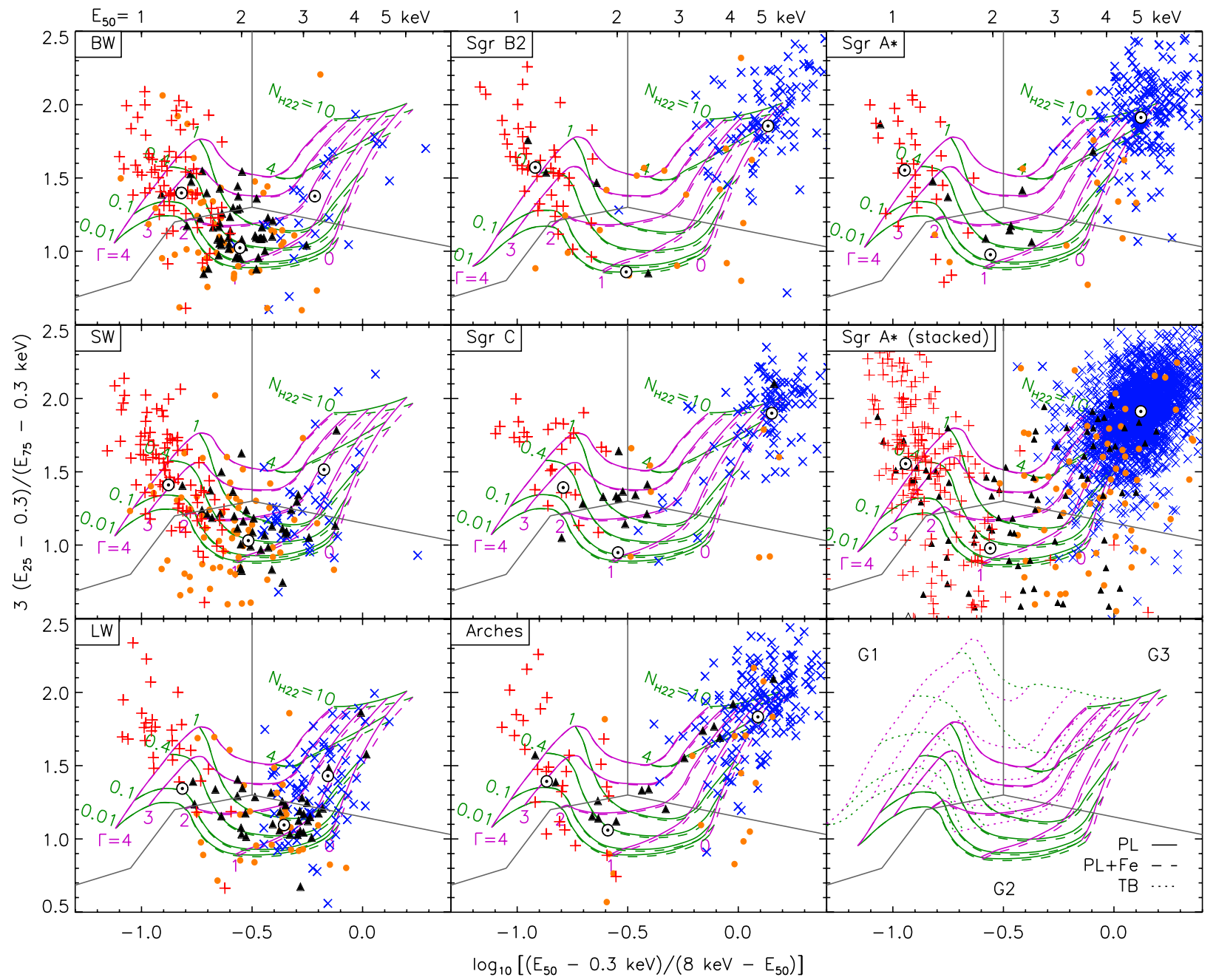

Figure 2. Quantile diagrams $(0.3-8 \mathrm{keV})$ of the X-ray sources with $\mathrm{S} / \mathrm{N} \geqslant 3$ in the GB fields overlaid with grids for a simple PL model (PL, solid lines, PL index $\Gamma$ $=0,1,2,3$, and $4, N_{\mathrm{H} 22}=0.01,0.1,0.4,1$, and 10), a PL plus an iron line model (PL+Fe, dashed, at $6.7 \mathrm{keV}$ with $0.4 \mathrm{keV} \mathrm{EW}$ ), and TB model (TB, dotted, $k T$ $=0.2,0.4,1,2,4$, and $10 \mathrm{keV}, N_{\mathrm{H} 22}=0.01,0.1,0.4,1$, and 10 , only shown in the bottom-right plot for clarity). The energy quantile $E_{x}$ corresponds to the energy below which $x \%$ of the counts are detected. The (red) crosses are for the relatively soft sources (S/N $\geqslant 3$ in $S_{C}$, but not in $H_{C}$ ), the (blue) "×"s for the hard sources ( $\mathrm{S} / \mathrm{N} \geqslant 3$ in $H_{C}$, but not in $S_{C}$ ), the (black) triangles for the bright sources ( $\mathrm{S} / \mathrm{N} \geqslant 3$ in both $S_{C}$ and $H_{C}$ ), and the (orange) dots for the faint sources ( $\mathrm{S} / \mathrm{N} \geqslant 3$ only in $\left.B_{C}\right)$. The (gray) lines from $(-0.5,1.3)$ divide each diagram into the soft $(G 1)$, medium $(G 2)$, and hard groups $(G 3)$. $\odot$ s mark the quantiles of stacked photons in each group.

(A color version of this figure is available in the online journal.)

on average, well separately from the foreground sources. We explore this more in Section 3.2.

The quantile diagrams nicely illustrate the spectral diversity of the X-ray sources in the GB fields, but poor photon statistics also contributes to the scatter. To reduce systematic errors caused by poor statistics in assigning spectral types while allowing the spectral diversity of the sources in each field, we divide the diagram into three groups as shown by the (gray) lines originating at $(-0.5,1.3)$. The left section represents most foreground thermal sources ( $G 1$ : soft group), the middle section most unabsorbed accreting sources ( $G 2$ : medium group), and the right section the absorbed thermal or accreting sources (G3: hard and absorbed group). The division between $G 1$ and $G 3$ is devised to be somewhat robust ${ }^{5}$ against variations in detector response between ACIS-I and ACIS-S (see H04; H05); or induced by gradual loss of low-energy response. The final results (e.g., $\log N-\log S$ distributions) are not sensitive to small changes of the group boundaries (e.g., shifting the boundaries

\footnotetext{
5 Under the PL model, the boundary of the $G 1$ and $G 2$ groups stays in roughly between $\Gamma=2$ and 3 .
}

by $\sim 0.1$ in $x$ or $y$ ). The mean quantiles for each group (marked by $\odot$ ) are calculated by the stacked photons of the sources in the group with $\mathrm{S} / \mathrm{N} \geqslant 3$ and net counts $\leqslant 1000$ (to avoid being dominated by a few bright sources) in $B_{X}$. For a given model class (e.g., PL), we estimate the spectral model parameters (e.g., $\Gamma$ and $N_{\mathrm{H}}$ ) of the sources in each group using the mean quantiles.

\subsection{Spectral Hardening versus Radial Offset from $G C$}

Table 3 summarizes the group mean quantiles of the $G 2$ and $G 3$ sources and corresponding model parameters under the PL and PL+Fe models. The $G 2$ sources in the high extinction fields are omitted in the table since they are mostly foreground sources. For comparison, the table also shows the model parameters estimated from spectral model fits. In order to increase photon statistics, we stacked the spectra of sources within a group with net counts $\leqslant 1000$ for spectral fitting ${ }^{6}$ and the spectra

\footnotetext{
6 One can use a spectral model fit on individual sources with net counts $>200-300$, but in order to establish more reliable statistics for the presence of the line emission for the group, we also stack moderately bright sources with net counts up to 1000 .
} 
Table 3

Spectral Model Parameters for the $G 2$ and $G 3$ Sources

\begin{tabular}{|c|c|c|c|c|c|c|c|c|c|c|}
\hline \multirow[t]{2}{*}{ Field } & \multirow[b]{2}{*}{$\begin{array}{c}E_{50} \\
(\mathrm{keV})\end{array}$} & \multirow[t]{2}{*}{$\begin{array}{l}\text { Quartile } \\
\text { Ratio }^{\mathrm{a}}\end{array}$} & \multicolumn{2}{|c|}{$\begin{array}{c}\text { PL from } \\
\text { Quantile Diagram }^{\mathrm{b}}\end{array}$} & \multicolumn{2}{|c|}{$\begin{array}{l}\mathrm{PL}+\mathrm{Fe}(\mathrm{He} \alpha) \text { from } \\
\text { Quantile Diagram }^{\mathrm{c}}\end{array}$} & \multicolumn{4}{|c|}{$\begin{array}{l}\mathrm{PL}(+\mathrm{Fe} \mathrm{He} \alpha) \text { from } \\
\text { Spectral Model Fit }^{\mathrm{d}}\end{array}$} \\
\hline & & & $\Gamma$ & $\begin{array}{c}N_{\mathrm{H} 22} \\
\left(\times 10^{22} \mathrm{~cm}^{-2}\right)\end{array}$ & $\Gamma$ & $\begin{array}{c}N_{\mathrm{H} 22} \\
\left(\times 10^{22} \mathrm{~cm}^{-2}\right)\end{array}$ & $\Gamma$ & $\begin{array}{c}N_{\mathrm{H} 22} \\
\left(\times 10^{22} \mathrm{~cm}^{-2}\right)\end{array}$ & $\begin{array}{l}\mathrm{EW}^{\mathrm{e}} \\
(\mathrm{keV})\end{array}$ & $\chi^{2} / \mathrm{DoF}^{\mathrm{f}}$ \\
\hline \multicolumn{11}{|c|}{ Unabsorbed hard sources $(G 2)$} \\
\hline BW & $1.97(2)$ & $1.02(1)$ & $\mathbf{1 . 3 8}(03)$ & $0.31(03)$ & $\mathbf{1 . 4 2}(03)$ & $0.32(05)$ & $\mathbf{1 . 3 6}(2)$ & $0.26(01)$ & $0.15(9)$ & $113.3 / 167$ \\
\hline SW & $2.10(5)$ & $1.03(2)$ & $\mathbf{1 . 3 5}(07)$ & $0.37(06)$ & $\mathbf{1 . 3 8}(10)$ & $0.38(08)$ & $1.22(3)$ & $0.25(03)$ & $-\mathrm{g}$ & $48.7 / 69$ \\
\hline$\underline{\mathrm{LW}}$ & $2.66(4)$ & $1.09(2)$ & $\mathbf{1 . 2 8}(07)$ & $0.73(09)$ & $\mathbf{1 . 3 5}(07)$ & $0.79(09)$ & $0.99(2)$ & $0.38(02)$ & $-\mathrm{g}$ & $125.9 / 148$ \\
\hline \multicolumn{11}{|c|}{ Absorbed hard sources (G3) } \\
\hline BW & $3.22(13)$ & $1.38(9)$ & $1.66(37)$ & $2.20(70)$ & $1.74(37)$ & $2.30(70)$ & $1.22(4)$ & $1.66(16)$ & $-\mathrm{g}$ & $24.8 / 26$ \\
\hline SW & $3.39(7)$ & $1.52(4)$ & $\mathbf{1 . 7 7}(23)$ & $2.90(50)$ & 1.91(23) & $3.10(50)$ & $\mathbf{1 . 5 8}(4)$ & $2.78(19)$ & $-\mathrm{g}$ & $35.4 / 22$ \\
\hline LW & $3.48(5)$ & $1.43(3)$ & 1.21(10) & $1.95(15)$ & $\mathbf{1 . 3 2}(10)$ & $2.10(20)$ & $\mathbf{1 . 3 0}(2)$ & $1.89(06)$ & $0.17(8)$ & $93.1 / 115$ \\
\hline Sgr B2 & $4.75(4)$ & $1.86(2)$ & $-0.37(14)$ & $3.40(80)$ & $0.25(17)$ & $5.70(90)$ & $0.50(1)$ & $6.20(22)$ & $0.61(7)$ & $105.2 / 154$ \\
\hline Sgr C & $4.81(3)$ & $1.90(2)$ & $-0.26(10)$ & $4.8(1.0)$ & $0.46(21)$ & $7.8(1.2)$ & $-\mathbf{0 . 1 0}(1)$ & $3.95(18)$ & $0.38(5)$ & $172.3 / 189$ \\
\hline Arches & $4.54(2)$ & $1.83(1)$ & $\mathbf{0 . 1 4}(07)$ & $4.00(50)$ & $0.67(14)$ & $5.70(70)$ & $\mathbf{0 . 8 5}(1)$ & $5.17(12)$ & $0.66(5)$ & $319.4 / 363$ \\
\hline Sgr A* & $4.69(2)$ & $1.91(1)$ & $\mathbf{0 . 3 1}(14)$ & $6.40(60)$ & $0.94(14)$ & $9.00(80)$ & $1.02(1)$ & $6.95(12)$ & $0.46(4)$ & $324.3 / 364$ \\
\hline
\end{tabular}

Notes. ${ }^{\text {a }} 3\left(E_{25}-0.3 \mathrm{keV}\right) /\left(E_{75}-0.3 \mathrm{keV}\right)$.

$\mathrm{b}$ The parameter estimates based on quantile analysis for a PL model.

c The same as (b) but with a fixed $0.4 \mathrm{keV} \mathrm{EW}$ at $6.7 \mathrm{keV}$.

$\mathrm{d}$ The parameter estimates by the spectral model fit.

${ }^{\text {e }}$ The EW of the $6.7 \mathrm{keV}$ line.

${ }^{\mathrm{f}}$ Degrees of freedom (DoF).

g Due to poor statistics, the spectral fit is done with a PL model without an iron line; see Section 4.

were binned to have at least 40 counts in each bin. The model used is a PL plus an iron emission line for which we have chosen the $6.7 \mathrm{keV} \mathrm{Fe} \mathrm{XXV} \mathrm{He} \alpha$ line because it has also been observed in the spectra of the X-ray point sources in the deep survey of the Sgr A* field (M03), the shallow survey of the GC strip (Wang et al. 2002), and other parts of the Galactic plane (Ebisawa et al. 2005). The $6.4 \mathrm{keV}$ neutral iron line is also present in some sources of the GB fields, but it is generally more prominent as unresolved diffuse emission (Wang et al. 2002). Note our aperture photometry is designed to minimize possible contamination of the diffuse emission through background subtraction (H05). We have chosen the $0.4 \mathrm{keV} \mathrm{EW}$ for the line in quantile analysis (and the $\log \mathrm{N}-$ $\log S$ distribution later) because it lies in the EW range estimated by spectral model fits on the $G 3$ sources and Muno et al. (2004) found a similar value $(\sim 0.4 \mathrm{keV})$ for the bright sources in the Sgr A* field.

Under the PL model, both groups show a trend of increasing hardness of the intrinsic spectra on an approach to GC (i.e., $\Gamma \gtrsim 1$ for the Window fields versus $\Gamma<1$ for other GB fields). This apparent trend can be attributed to a few factors.

In the case of the $G 2$ sources in the Window fields, the group contains a large number of foreground coronal sources and a background active galactic nucleus (AGN) in addition to the GB sources. For instance, in the BW and SW, about $70 \%$ of the sources with $\mathrm{S} / \mathrm{N} \geqslant 3$ in the $H_{C}$ band are the background AGN (see Figure 5). Therefore, the group is perhaps too contaminated for the apparent trend to be taken for real.

The similar trend in the $G 3$ group appears to be more realistic. However, comparing the PL+Fe model grid with the PL model grid in the quantile diagram suggests this trend can be an artifact of using the PL model at least in part. Indeed the trend is alleviated under the PL+Fe model as shown by the model parameters estimated by both quantile analysis and the spectral fits (Table 3). Poor statistics in the $G 3$ sources of the BW and SW does not allow any meaningful constraint of the iron line emission in the spectra, but the stacked spectrum of the $G 3$

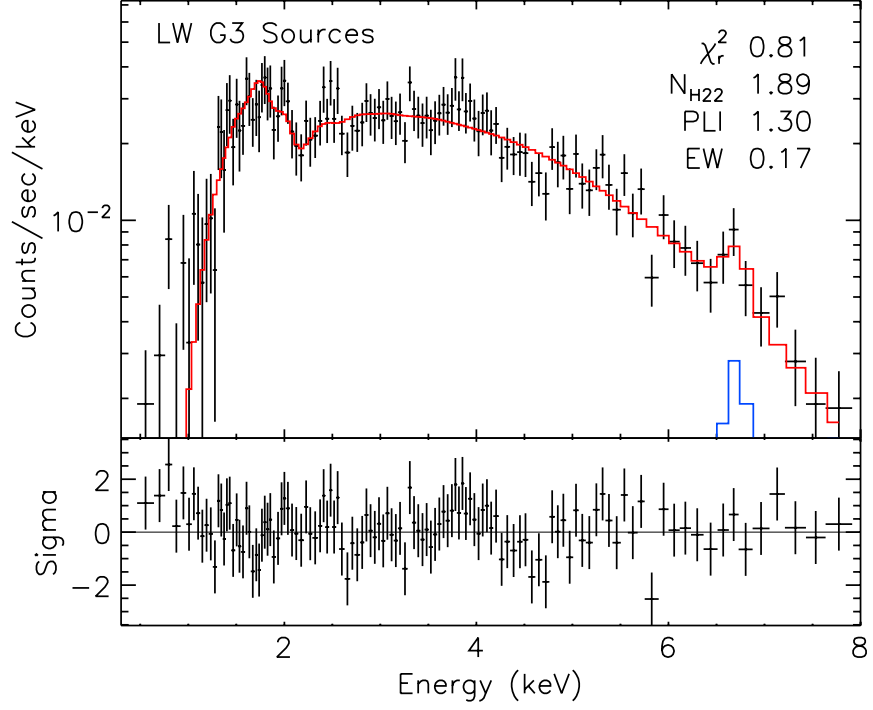

Figure 3. Stacked spectra of the $G 3$ sources (net counts <1000) in the LW with the PL+Fe XXV He $\alpha$ (6.7 keV line) fit. The estimated EW of the line is $0.17 \pm 0.08 \mathrm{keV}$.

(A color version of this figure is available in the online journal.)

sources in the LW does show a clear hint of the $6.7 \mathrm{keV}$ line (Figure 3; see also Revnivtsev et al. 2009). The relatively weak line feature $(\mathrm{EW} \sim 0.17 \mathrm{keV})$ in the $\mathrm{LW}$ can be explained by the relatively large contribution of the $\mathrm{AGN}$ in the group compared to other GB fields (see Figure 5).

With the inclusion of a $6.7 \mathrm{keV}$ line, the PL index $(\Gamma)$ becomes largely consistent across the GB fields with the possible exception of the Sgr B2 or C field (see Section 5.4). The result indicates that the galactic X-ray sources in the LW field may be the same type of sources as seen in the other GB fields closer to the GC. If this is true, the GB X-ray sources indeed extend out to at least 1.4 from the GC. 

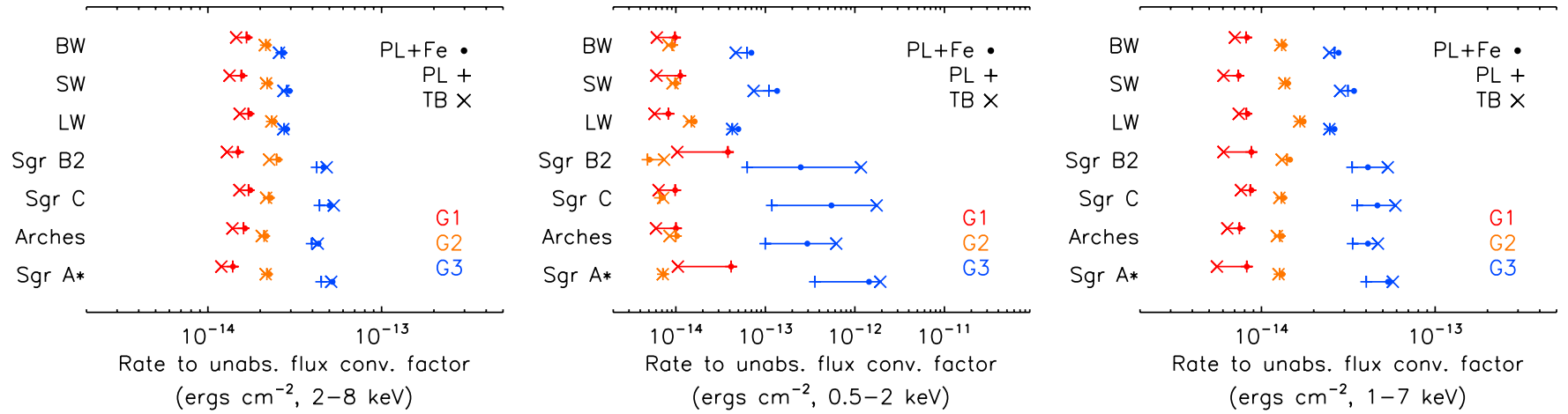

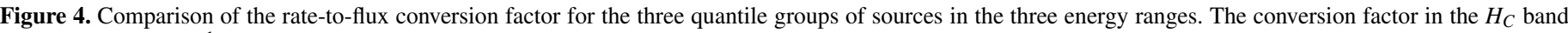

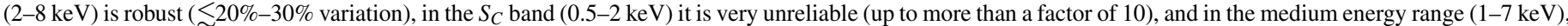
there are significant variations (up to $\sim 100 \%$ ).

(A color version of this figure is available in the online journal.)
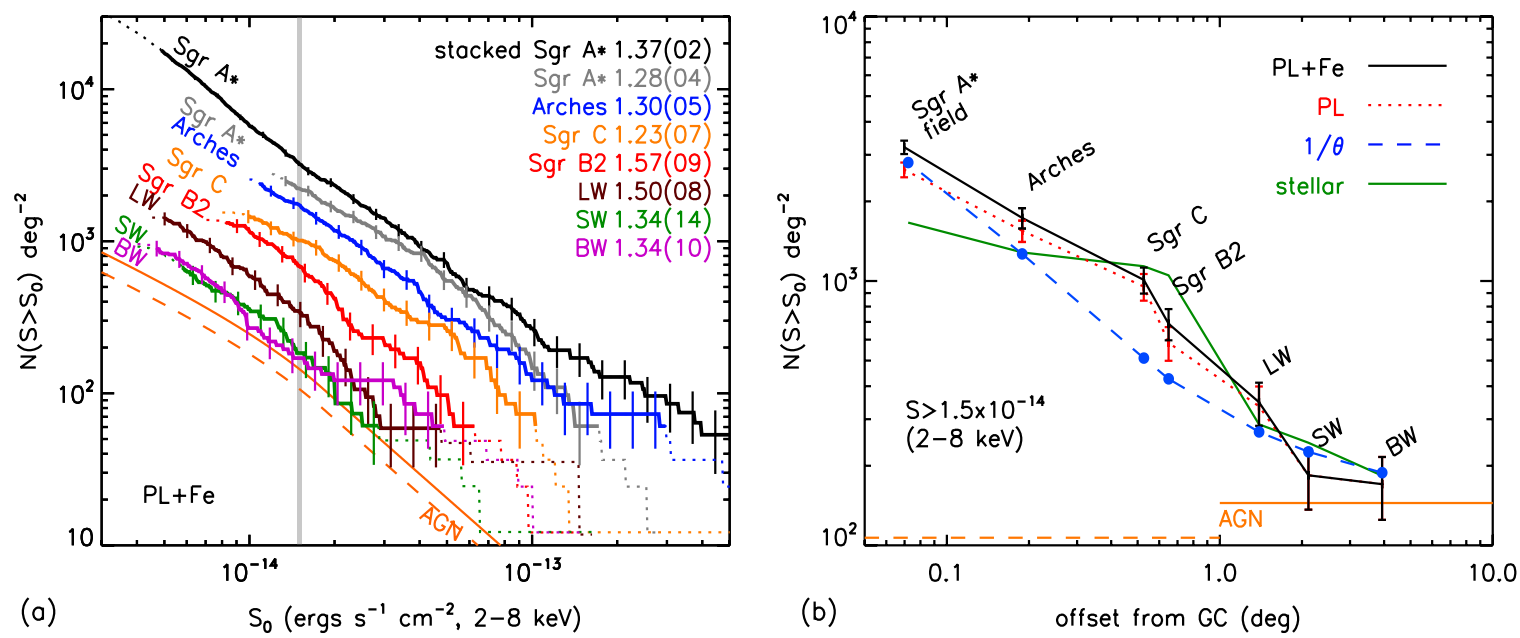

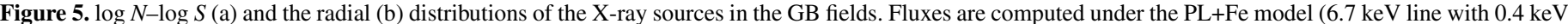

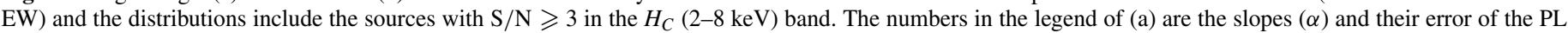

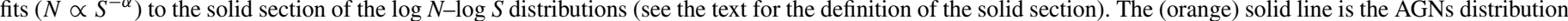

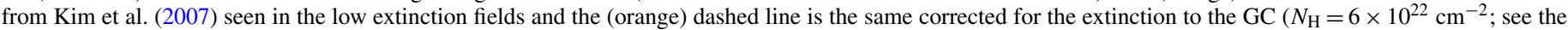

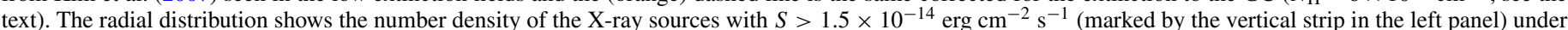

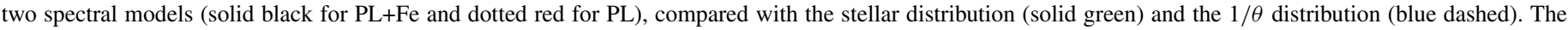
$x$-axis in the radial distribution is the average offset value of the sources in each field.

(A color version of this figure is available in the online journal.)

\subsection{Flux Estimates}

Based on the group model parameters, we estimate the source flux in the conventional energy bands, using the instrument response files at the aim point and scaling them for source position on the detector by the exposure map (H05). ${ }^{7}$ For the stacked data, we use the average response files weighted by the exposure of each observation.

The quantile diagram can assign the model parameters (e.g., $\Gamma=1.7$ versus 1.0$)$ appropriate to a given model class for the sources, but it cannot determine which model class (e.g., PL versus TB) is right for the sources. A certain model can only be ruled out when the derived values of the parameters are unphysical or with external information (e.g., optical identifications). In order to estimate the systematic errors arising from the improper choice of the model class, we compare flux estimates under three different model classes: PL, PL+Fe, and TB. In order to see the significance of the difference among these models, Figure 4 compares the conversion factor of count rate

\footnotetext{
7 The latest CIAO tools (version 3.4 or higher) can calculate the response files appropriate for each source location.
}

to unabsorbed flux for sources near the aim point in each group under the three model classes. In the case of the $H_{C}$ band, the difference between the model classes is very small, but in the $S_{C}$ band, the conversion factor can differ by more than a factor of 10 . We take the largest difference in the flux estimates among the three model classes as the systematic errors $\left(\sigma_{s}\right)$ and compare them with the pure count-based statistical errors $\left(\sigma_{c}\right)$. For the flux estimates in the $H_{C}$ band, $\sigma_{s} \sim 20 \%-30 \%$ and we get $\sigma_{s}<\sigma_{c}$ for $\sim 87 \%$ of the sources with $\mathrm{S} / \mathrm{N} \geqslant 3$, and $\sigma_{s}<\sigma_{c}$ for $\sim 62 \%$ even with $\mathrm{S} / \mathrm{N} \geqslant 5$. In the $S_{C}$ band, $\sigma_{s}$ can be larger than $1000 \%$, and we get $\sigma_{s}<\sigma_{c}$ only for $28 \%$ with $\mathrm{S} / \mathrm{N} \geqslant 3$ and for $16 \%$ with $\mathrm{S} / \mathrm{N} \geqslant 5$.

This exercise does not explore all the possible model classes, but the results indicate the $H_{C}$ band flux estimates in this method are robust and relatively insensitive to the choice of the model classes. However, the $S_{C}$ band flux estimates can be dominated by the systematic errors arising from improper selection of the spectral model. The fundamental difference between the $S_{C}$ and the $H_{C}$ band is that the $S_{C}$ band is very sensitive to the range of interstellar absorption in the GB fields, $\sim 10^{21-23} \mathrm{~cm}^{-2}$, while the $H_{C}$ band is not. In the following, we limit our discussion to 
the $H_{C}$ band results using the sources with $\mathrm{S} / \mathrm{N} \geqslant 3$ in $H_{C}$ (" $\times$ "s and triangles in Figure 2), which are likely to be GB sources (and AGN) rather than the foreground sources; see also van den Berg et al. (2009) for the spectral choices for the flux estimates in $B_{X}$. If the number of counts in the stacked spectrum of a quantile group $(G 1, G 2$, or $G 3)$ were large enough, a spectral fit would be better suited for determining the underlying spectral model and its parameters, but a fit can also leave ambiguity over the correct spectral model. Since in the $H_{C}$ band the difference driven by the model class is less significant than the statistical errors, we simply use the model parameters estimated by quantile analysis.

\section{SOURCE DISTRIBUTION}

\subsection{Eddington and Malmquist Biases}

In order to explore the effect of the Eddington bias (EB), which makes the faintest sources appear brighter than they really are, we simulate three spectral types of sources (one for each group) based on the group mean quantiles for each field. Using the MARX simulation code, we generate the sources with net counts $\left(B_{X}\right)$ from 5 to 400 , using an $S^{-1}$ distribution to cover the wide count range efficiently. We scatter 200-250 of these sources randomly over the real events and apply the regular analysis procedure. We repeat the procedure 1000 times. The fake sources are not allowed to overlap each other but they can fall on top of the real sources. The results indicate that the EB is noticeable in the sources with $<10$ counts, which can appear as bright as 15-20 count sources, depending on the field (or up to $\sim 30$ count sources for the stacked Sgr A* field). Since we consider sources with $\mathrm{S} / \mathrm{N} \geqslant 3$ in the $H_{C}$ band, which corresponds to $\gtrsim 16-20$ counts at least ( $\gtrsim 30$ counts for the stacked Sgr A* field), we expect that the EB is not a major contributor to the errors of the following distributions.

The Malmquist bias (MB) is due to the exposure-dependent volume (depth) coverage. The MB is usually a concern for luminosity distributions but not for $\log N-\log S$ distributions in the apparent (detected) flux space. However, the $\log N-\log S$ distributions in the unabsorbed flux space can be subject to the MB when strong interstellar absorption limits the depth of the view, underestimating the true distribution. Therefore, the faint end of the $\log N-\log S$ distribution can be lower than the true distribution and so the MB counteracts the EB to some extent.

With $\geqslant 100 \mathrm{ks}$ exposure, all the sources with an unabsorbed flux $\gtrsim 10^{-14}$ erg cm$~^{-2} \mathrm{~s}^{-1}$ can be detected at the far side of the Galaxy with $\mathrm{S} / \mathrm{N} \geqslant 3$ in $H_{C}$ under the assumption of the total integrated absorption ${ }^{8}$ of $N_{\mathrm{H}} \sim 12 \times 10^{22} \mathrm{~cm}^{-2}$. Therefore, the $\mathrm{MB}$ is not a concern for sources with $\gtrsim 10^{-14} \mathrm{erg} \mathrm{\textrm {cm } ^ { - 2 }}$ $\mathrm{s}^{-1}$ (or $\gtrsim 5 \times 10^{-15} \mathrm{erg} \mathrm{cm}^{-2} \mathrm{~s}^{-1}$ for the Window fields) under the assumption of a PL with $\Gamma=1.0$ for the X-ray spectrum. This does not mean we can access X-ray sources of a certain luminosity uniformly all the way through the Galaxy. For instance, the unabsorbed flux of $S>10^{-14} \mathrm{erg} \mathrm{cm}^{-2} \mathrm{~s}^{-1}$ corresponds to $L_{X} \gtrsim 8 \times 10^{31} \mathrm{erg} \mathrm{s}^{-1}$ at the GC $(8 \mathrm{kpc}$, $N_{\mathrm{H}} \sim 6 \times 10^{22} \mathrm{~cm}^{-2}$ ) and $L_{X} \gtrsim 7 \times 10^{32} \mathrm{erg} \mathrm{s}^{-1}$ at $20 \mathrm{kpc}$ $\left(N_{\mathrm{H}} \sim 12 \times 10^{22} \mathrm{~cm}^{-2}\right)$. The situation is a bit more tricky since under the quantile group method we assign fixed spectral parameters with a fixed $N_{\mathrm{H}}$ value for the X-ray spectra of all the sources in each group, which in fact have a diverse $N_{\mathrm{H}}$ distribution (e.g., the $G 3$ group in the $\mathrm{Sgr} \mathrm{A}^{*}$ field). However, the sources with an unabsorbed flux of $S>10^{-14} \mathrm{erg} \mathrm{cm}^{-2}$

\footnotetext{
8 Assuming the absorption to the GC to be $6 \times 10^{22} \mathrm{~cm}^{-2}$ (Baganoff et al.
} 2003 ) and the symmetry with respect to the GC. $\mathrm{s}^{-1}$ in this method are free of the MB for $\gtrsim 100 \mathrm{ks}$ exposure. The MB can be a problem for the $G 1$ and $G 2$ sources in the high extinction fields, but their contribution in the $\log N-\log S$ distribution of the $H_{C}$ band is negligible compared to the $G 3$ sources.

\subsection{Sky Coverage}

For the $\log N-\log S$ distribution, we need to know the sky coverage as a function of flux. In order to minimize the systematic errors associated with spectral-type assignment, we calculate the sky coverage of each source based on the detected photon counts in the three energy bands as follows (Cappelluti et al. 2005; H05). For each observation, we generate the background-only images by removing the counts in the source regions in the image and filling the region with the counts using the statistics in the surrounding regions (dmfilth). ${ }^{9}$ At every pixel in the background images, we calculate the minimum source counts required for detection with $S / N \geqslant 3$. For the sky coverage of a given source, we take the sky area where the minimum counts are less than the net counts of the source in the band. These sky coverage values agree well with those expected from the simulated sources of three spectral types using the MARX (Section 4.1). On average, they are within $10 \%$ for the cases with $S / N \geqslant 3$, which indicates this method accounts for the completeness as well.

\subsection{The $\log N-\log S$ and Radial Distributions}

The $\log N-\log S$ and radial distributions of the X-ray sources in the GB fields are shown in Figures 5(a) and (b), respectively. The $\log N-\log S$ distribution was computed using sources with $\mathrm{S} / \mathrm{N} \geqslant 3$ in the $H_{C}$ band, under both the PL and PL+Fe models described in Section 3, with the latter result plotted in Figure 5(a). The source number density values plotted against angular distance from $\mathrm{Sgr} \mathrm{A}^{*}$ in Figure 5(b) are projected from the $\log N-\log S$ distributions at the flux value indicated by the vertical gray line $\left(S>S_{0}=1.5 \times 10^{-14} \mathrm{erg} \mathrm{cm}^{-2}\right.$ $\mathrm{s}^{-1}$ ) in Figure 5(a). As seen in Figure 5(b), the total source densities under the PL model are slightly lower than the same under the PL+Fe model in the high extinction fields, while both distributions under these models are nearly identical in the three low extinction Window fields.

For clarity, we define the statistically robust section of each distribution in Figure 5(a) and emphasize it with a solid line. This "solid section" is defined to contain contributions from at least 10 or more sources, which set the upper limit of the range (e.g., $S_{0} \sim 10^{-13} \mathrm{erg} \mathrm{cm}^{-2} \mathrm{~s}^{-1}$ for $\mathrm{Sgr} \mathrm{C}$ ). The lower limit is set by the flux value at which the sky coverage of the contributing $G 3$ sources is greater than $50 \%$ of the maximum sky coverage, i.e., the full field of view (FoV; e.g., $S_{0} \sim 10^{-14} \mathrm{erg} \mathrm{cm}^{-2} \mathrm{~s}^{-1}$ for Sgr C). In this way, we avoid the statistical bias or fluctuation due to either low source statistics at the bright end of the accessible flux range or limited sky coverage at the faint end of the range. The portion of each distribution not meeting the above criteria is dotted.

The slope $(\alpha)$ of the $\log N-\log S$ distribution is calculated by a PL fit $\left(N \propto S^{-\alpha}\right)$ to the solid line section of the distribution. The $y$-axis error of the distribution is given by the quadratic sum of the statistical error (shown in the figure) and a constant systematic error $(\sim 20 \%$, the difference between the PL and the PL+Fe model). As expected for the narrow FoV of ACIS-I

\footnotetext{
9 http://cxc.harvard.edu/ciao/ahelp/dmfilth.html
} 
observations, the slopes of the $\log N-\log S$ distributions are largely consistent with the -1.5 slope within $\sim 2 \sigma$ except for the stacked Sgr A* field, which shows a hint of the actual deviation $(\sim 6 \sigma)$ from the -1.5 slope. Note the calculated slopes are only for guiding purpose, and they should not be taken seriously for representing the population since a simple PL does not fit some of the distributions very well.

The AGN distribution is taken from Kim et al. (2007), using a PL model with $\Gamma=1.7$ for the X-ray spectra. The (orange) dashed line indicates the reduced AGN population that can be seen through the high extinction fields such as the Sgr A* field, since the unabsorbed flux is corrected for the average absorption of the X-ray sources, mostly Galactic and centered around the GC, which should be about half of the total absorption for the AGN. For simplicity, we correct another $N_{\mathrm{H}}=6 \times 10^{22} \mathrm{~cm}^{-2}$ for the AGN seen in the high extinction fields.

For the Sgr A* field, we plot the results from both the stacked data (black) and the $100 \mathrm{ks}$ exposure (gray) in Figure 5(a) and use only the stacked data in Figure 5(b). The spectral models from the $100 \mathrm{ks}$ exposure are used for both data sets for fair comparison with other fields and to avoid any spectral parameter driven variations between two exposures for the $\mathrm{Sgr}$ A* field. The distribution of the stacked data is $\sim 40 \%$ higher at $S_{0}=10^{-14} \mathrm{erg} \mathrm{cm}^{-2} \mathrm{~s}^{-1}$ than the same for the $100 \mathrm{ks}$ exposure. A few factors such as the $\mathrm{MB}^{10}$ are responsible for the difference, but the main cause of the difference is suspected to be the $\mathrm{X}$ ray variability of the sources. The stacked data $(750 \mathrm{ks})$ simply have a better chance of detecting the sources or catching high flux states of the sources than for the shorter exposure (100 ks). For instance, the 20 brightest sources in the $H_{C}$ band in the $100 \mathrm{ks}$ observation of the $\mathrm{Sgr} \mathrm{A}^{*}$ field are found to be about $30 \%$ brighter on average in the stacked data set, and five of the 20 brightest sources in the stacked data were not detected in the $100 \mathrm{ks}$ observation. This variation qualitatively agrees with the change seen in the $\log N-\log S$ distributions, but the diverse nature of the X-ray variability and duty cycles makes it hard to quantify the resulting difference in the $\log N-\log S$ distributions.

The radial distribution is generated from the sources with $S>S_{0}=1.5 \times 10^{-14} \mathrm{erg} \mathrm{cm}^{-2} \mathrm{~s}^{-1}$, plotted over the stellar distribution (green) and the $1 / \theta$ distribution (blue dashed). Both the stellar and $1 / \theta$ distributions are averaged over the ACIS-I FoV $\left(17^{\prime} \times 17^{\prime}\right)$ of the GB fields. The $S_{0}$ value for the radial distribution is chosen as a compromise between having sufficient source statistics in the Window fields $\left(S_{0} \lesssim 2 \times 10^{-14} \mathrm{erg} \mathrm{cm}^{-2}\right.$ $\mathrm{s}^{-1}$ ) and avoiding statistical biases in the high extinction fields $\left(S_{0} \gtrsim 10^{-14} \mathrm{erg} \mathrm{cm}^{-2} \mathrm{~s}^{-1}\right)$. The curve resulting under the PL+Fe model (black solid) is more centrally concentrated around the GC than the PL model (red dotted).

The stellar distribution is derived from the Galactic stellar models compiled by M06, and it consists of a central spherical cluster $\left(\rho_{A}\right.$; Equation (1) in M06), a central disk $\left(\rho_{B}\right.$; Equation (2) in M06), a triaxial ellipsoidal GB ( $\rho_{C}$; Equation (3) in M06) and a Galactic disk ( $\rho_{D}$, Equation (6) in M06; for the origin of the formulae, see also Launhardt et al. 2002, hereafter L02; Kent et al. 1991, hereafter K91). For the first three components $\left(\rho_{A}, \rho_{B}\right.$, and $\left.\rho_{C}\right)$, we use the formula and the parameter values in L02 and M06. For the Galactic disk component $\left(\rho_{D}\right)$, M06 use a simple exponential form in K91 and employ $10^{11} M_{\odot}$ for the total Galactic disk mass for the overall normalization, but since the first three components are mainly for the stellar mass,

${ }_{10}$ Note that the unstacked Sgr A* field (Obs. ID 3665) has the shortest GTI (88 ks) among the seven fields. we believe this is an overestimate. Therefore, we use a normalization that matches the local stellar mass density of 0.044 $M_{\odot} \mathrm{pc}^{-3}$ (Robin et al. 2003). This gives $1.8 \times 10^{10} M_{\odot}$ for the whole disk, which is roughly consistent with the estimate by Robin et al. $\left(2003 ; 2.2 \times 10^{10} M_{\odot}\right) .{ }^{11}$ Since we expect both the X-ray and stellar sources are centrally concentrated around the GC, in Figure 5(b) we further assume that all detected hard Galactic X-ray sources are at a distance of 6-10 kpc, which is justified given that the stellar models predict that $\gtrsim 80 \%$ of sources along the line of sight of the GB fields lie in the same distance range. Note the central concentration also makes our normalization change of the Galactic disk component less important in the outcome, but we find that the change makes this Galactic stellar mass model consistent with other Galactic stellar number density models (see Section 5.1 and Table 4). These stellar model components have about a factor of 2 uncertainty (M06; L02).

The normalization of the stellar and $1 / \theta$ distributions is set by a simple $\chi^{2}$ fit to the radial-distribution curve under the PL+Fe model (Figure 5(b)). We use the stacked result for the Sgr A* field. The radial distribution shows the GB X-ray sources are highly concentrated at the GC, more than the stellar distribution. It also shows that the hard GB X-ray sources extend out to $>1.4$ from the $\mathrm{GC}$, roughly following an empirical relation of $1 / \theta$ with some excess in the Arches Cluster, Sgr C, and Sgr B2 fields.

The excess of the X-ray source to stellar distribution near the GC does not appear as prominent if we use the $100 \mathrm{ks}$ exposure of the $\mathrm{Sgr}$ A* field at $S_{0}=1.5 \times 10^{-14} \mathrm{erg} \mathrm{cm}^{-2} \mathrm{~s}^{-1}$. However, the trend of the relative excess of the X-ray sources toward the GC is present from the Sgr B2 to the Sgr A* field (e.g., the Sgr B2 field has a deficit under the current relative normalization in Figure 5(b)), and the $\log N-\log S$ distributions of the $100 \mathrm{ks}$ exposure and the stacked Sgr $A^{*}$ field become more consistent at $S_{0} \gtrsim 2 \times 10^{-14} \mathrm{erg} \mathrm{cm}^{-2} \mathrm{~s}^{-1}$. Therefore, the excess of X-ray sources toward the GC with respect to the considered stellar model appears real. We explore this excess in more detail in Section 5.1 .

\section{DISCUSSION}

We find that the number density of the hard X-ray sources in the GB is significantly elevated above the AGN density out to at least the LW at 1.4 separation from Sgr A* (Figure 5(a)). Furthermore, although empirical (see Figure 6 for the composition of the stellar population), the radial distribution of the hard X-ray sources roughly follows a $1 / \theta$ relation out to this field (Figure 5(b)). This discovery suggests that all such sources observed within at least $\sim 200 \mathrm{pc}$ of the GC belong to the same centrally concentrated population. The similarity of the stacked spectra of the hard X-ray sources in all fields from Sgr A* to the $\mathrm{LW}$, and in particular the presence of a $6.7 \mathrm{keV}$ iron emission line strengthens our conclusion that a single underlying class of sources makes up this population.

\subsection{X-ray Source Density versus CV Density}

The current leading candidate to explain the X-ray sources within $20 \mathrm{pc}$ around the GC is magnetic CVs or intermediate polars (IPs) in particular (M04; L05). Recent population synthesis models by Ruiter et al. (2006, hereafter R06) show

\footnotetext{
11 The small difference is mainly due to the difference in the assumption of the distance to the GC: $8 \mathrm{kpc}$ for the model used here, and $8.5 \mathrm{kpc}$ for Robin et al. (2003).
} 
Table 4

X-ray Source and Stellar Density

\begin{tabular}{|c|c|c|c|c|c|c|c|c|}
\hline \multirow[t]{2}{*}{ Field } & \multicolumn{2}{|c|}{ X-ray Source ${ }^{b}$} & \multicolumn{5}{|c|}{ Stellar Model A ${ }^{\mathrm{c}}$} & \multirow{2}{*}{$\begin{array}{c}\text { Stellar Model B } \\
\text { Star Volume } \\
\text { Density } \\
\left(\mathrm{pc}^{-3}\right)\end{array}$} \\
\hline & $\begin{array}{l}\text { Surface } \\
\text { Density } \\
\left(\operatorname{deg}^{-2}\right)\end{array}$ & $\begin{array}{c}\text { Volume } \\
\text { Density } \\
\left(10^{-7} \mathrm{pc}^{-3}\right)\end{array}$ & $\begin{array}{c}\text { Star Volume } \\
\text { Density } \\
\left(\mathrm{pc}^{-3}\right)\end{array}$ & $\begin{array}{c}\text { Star Surface } \\
\text { Mass Density } \\
\left(10^{8} M_{\odot} \mathrm{deg}^{-2}\right)\end{array}$ & $\begin{array}{c}\text { X-ray to } \\
\text { Stellar Mass } \\
\left(10^{-7} M_{\odot}^{-1}\right)\end{array}$ & $\begin{array}{l}\text { X-ray } \\
\text { to Stars } \\
\left(10^{-7}\right)\end{array}$ & $\begin{array}{c}\text { Required CV } \\
\text { to Stars } \\
\left(10^{-5}\right)\end{array}$ & \\
\hline \multicolumn{9}{|c|}{ Field to field comparison } \\
\hline BW & $25(45)$ & $3.2(5.7)$ & 2.1 & 0.5 & $5.1(9.1)$ & $1.6(2.8)$ & $2.6(4.6)$ & 3.2 \\
\hline SW & $39(47)$ & $4.9(6.0)$ & 5.3 & 1.3 & $3.1(3.7)$ & $0.9(1.1)$ & $1.6(1.9)$ & 6.7 \\
\hline LW & $203(64)$ & $25(8.1)$ & 7.6 & 1.8 & $11(3.5)$ & $3.4(1.1)$ & $5.6(1.8)$ & 8.3 \\
\hline Sgr B2 & $582(93)$ & $73(11)$ & 41 & 10 & $5.7(0.9)$ & $1.8(0.3)$ & $2.9(0.5)$ & 8.8 \\
\hline Sgr C & $902(113)$ & $113(14)$ & 45 & 11 & $8.1(1.0)$ & $2.5(0.3)$ & $4.1(0.5)$ & 8.9 \\
\hline Arches & $1624(153)$ & 204(19) & 52 & 13 & $12(1.2)$ & $3.9(0.4)$ & $6.5(0.6)$ & 9.4 \\
\hline Stacked Sgr A* & $3100(187)$ & $389(23)$ & 68 & 17 & $18(1.1)$ & $5.7(0.3)$ & $9.5(0.6)$ & 9.1 \\
\hline $100 \mathrm{ksSgr} \mathrm{A}^{*}$ & $2117(176)$ & $266(22)$ & 68 & 17 & $12(1.1)$ & $3.9(0.3)$ & $6.5(0.5)$ & 9.4 \\
\hline \multicolumn{9}{|c|}{ By the fit to the radial distribution ${ }^{f}$} \\
\hline With stacked Sg & $*$ (Figure 5( & or Figure 6(a)) & & & & $10.3(0.5)$ & $3.2(0.2)$ & $5.3(0.3)$ \\
\hline With 100ks Sgr & (Figure $6(\mathrm{~b}$ & & & & & $9.2(0.5)$ & $2.8(0.2)$ & $4.7(0.3)$ \\
\hline \multicolumn{9}{|c|}{ By the fit with the freed relative normalization of $\rho_{A} \mathrm{~g}$} \\
\hline \multicolumn{6}{|c|}{ With stacked Sgr A* (Figure 6(c)) } & $7.1(0.6)$ & $2.2(0.2)$ & $3.6(0.3)$ \\
\hline \multicolumn{6}{|c|}{ With 100ks Sgr A* (Figure 6(d)) } & $7.5(0.6)$ & $2.3(0.2)$ & $3.8(0.3)$ \\
\hline
\end{tabular}

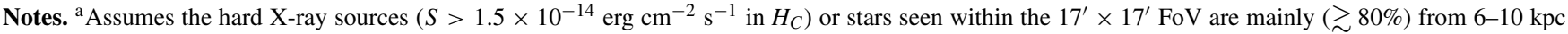
distance.

${ }^{\mathrm{b}}$ Using the PL+Fe model, we subtract the expected AGN numbers, $145 \mathrm{deg}^{-2}$ from the Window fields and $107 \mathrm{deg}^{-2}$ from the high extinction fields.

c The composite model in M06 and references therein. The model gives the stellar mass density in the unit of $M_{\odot}$ pc ${ }^{-3}$, and we assume the local value of 0.144 stars pc $^{-3}$ and $0.04 M_{\odot} \mathrm{pc}^{-3}$ to get the star number density (Robin et al. 2003). This relation should be good for the bulge in the case of CVs and active binaries, but perhaps not good for young stars (Sazonov et al. 2006).

d The stellar density model by Picaud \& Robin (2004) for the Galactic disk and outer GB. The model does not include a central nucleus, so the values for the Sgr B2, Sgr C, Arches, and Sgr A* fields are not reliable; see Section 5.1.

e The required CV to star density to explain the hard GB X-ray sources by IPs. We assume that IPs are 5\% of all CVs (e.g., $2 \%-8 \%$ in R06) and that about $12 \%$ of them are detected above $10^{32} \mathrm{erg} \mathrm{s}^{-1}$ (e.g., $\sim 10 \%-16 \%$ in R06), which corresponds $\sim 1.5 \times 10^{-14} \mathrm{erg} \mathrm{cm}^{-2} \mathrm{~s}^{-1}$ for the sources near the $\mathrm{GC}$ (see the text).

f The relative normalizations among stellar model components are fixed as given in Section 4.3.

$\mathrm{g}$ The relative normalization of the central spherical cluster component of the stellar distribution is allowed as a free parameter and fitted as well.

that IPs can constitute the majority of these X-ray sources under the assumption that IPs span a luminosity range of $\sim 3 \times 10^{29}-5 \times 10^{33} \mathrm{erg} \mathrm{s}^{-1}$ and that they make up $\sim 2 \%-8 \%$ of all CVs (see also M06 for a review of the population synthesis models for the X-ray sources in the GB). Now our radial distribution indicates this source population extends out to $\sim 200 \mathrm{pc}$ and the hard X-ray spectra with the iron emission line supports the idea that IPs are the major component of the population.

In this section, we compare the observed X-ray source density with the stellar (mass) density to see if CVs, especially IPs, can explain the majority of the detected X-ray sources. Table 4 summarizes the number density of the X-ray sources with $S>1.5 \times 10^{-14} \mathrm{erg} \mathrm{cm}^{-2} \mathrm{~s}^{-1}$ in the $H_{C}$ band and compares them with the stellar (mass) density. For the GB X-ray source densities, we subtract the expected number of the AGN, which is $145 \mathrm{deg}^{-2}$ in the Window fields and $107 \mathrm{deg}^{-2}$ in the high extinction fields, from the surface density (see Section 4.3; Kim et al. 2007).

Table 4 quotes the average stellar density over the volume defined by the distance between 6 and $10 \mathrm{kpc}$ in the $17^{\prime} \times 17^{\prime}$ FoV using two stellar models. Stellar model A is the same stellar mass model used in Figure 5 (M06; L02; K91). Since model A provides the stellar mass density, we use a local value of 0.144 stars $\mathrm{pc}^{-3}$ and $0.044 M_{\odot} \mathrm{pc}^{-3}$ to convert the mass density to the number density or vice versa (Picaud \& Robin 2004; Robin et al. 2003). As a consistency check, we also compute the stellar density using another stellar model (model B) by Picaud \& Robin (2004), which consists of a Galactic disk and a GB. This model describes the stellar number density in the outer GB and the Galactic disk. So it is properly normalized at the local Galaxy

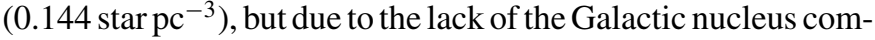
ponents $\left(\rho_{A}\right.$ and $\left.\rho_{B}\right)$, the stellar density for the GB fields within $1^{\circ}$ of the GC is underestimated. The two models agree within $30 \%$ for the Window fields where Galactic nucleus components are relatively unimportant. In the following, we use model A for comparing the X-ray source density with the stellar density.

The relative $\mathrm{X}$-ray source to stellar mass densities in the seven GB fields are $(0.3-1.8) \times 10^{-6} \mathrm{X}$-ray sources $M_{\odot}^{-1}$ at $S>1.5 \times 10^{-14} \mathrm{erg} \mathrm{cm}^{-2} \mathrm{~s}^{-1}\left(1.1 \times 10^{32} \mathrm{erg} \mathrm{s}^{-1}\right.$ for sources at the $\mathrm{GC}, 8 \mathrm{kpc})$. The large variation of the relative density among the seven fields reflects the mismatch between the X-ray and stellar distributions - the X-ray sources are more centrally concentrated than the stellar sources. The relative X-ray source to stellar number densities are $(0.9-5.7) \times 10^{-7} \mathrm{X}$-ray sources star $^{-1}$ at $S>1.5 \times 10^{-14} \mathrm{erg} \mathrm{cm}^{-2} \mathrm{~s}^{-1}$, depending on the field.

Now we assume IPs are $5 \%$ of all CVs (e.g., $\sim 2 \%-8 \%$ for the models in R06) and about $12 \%$ of IPs have the X-ray luminosity above $10^{32} \mathrm{erg} \mathrm{s}^{-1}$ (e.g., $\sim 10 \%-16 \%$ in R06; see also Heinke et al. 2008). Then the required $\mathrm{CV}$ to stellar density to explain the hard X-ray GB sources ranges from 1.6 to $9.5 \times 10^{-5}$ depending on the fields. If we assume a local star density of $0.144 \mathrm{pc}^{-3}$, these correspond to the equivalent local CV density of $(0.2-1.4) \times 10^{-5} \mathrm{pc}^{-3}$. Considering the current local CV density estimates $\left((1-3) \times 10^{-5} \mathrm{pc}^{-3}\right)$ in the literature (see, e.g., Ak et al. 2008; Grindlay et al. 2005; Pretorius et al. 2007), this result indicates that IPs can be the major component of 

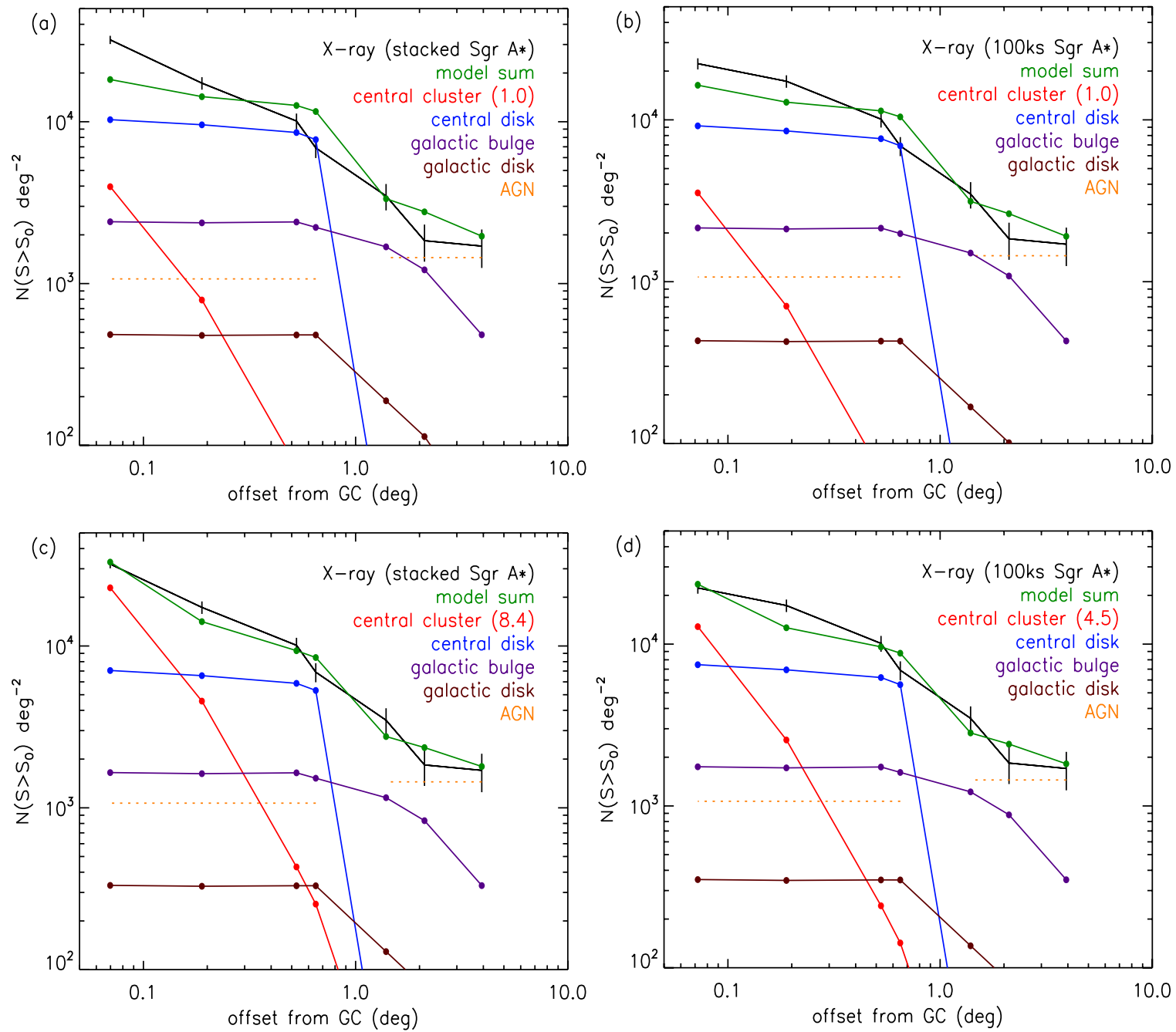

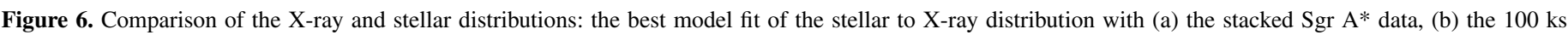

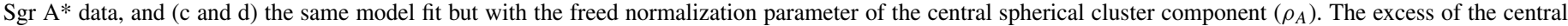
spherical cluster component $\left(\rho_{A}\right)$ needed for the best model fit is 8.4 or $4.5 \times$ higher than the original model, depending on the Sgr $\mathrm{A}^{*}$ data set.

(A color version of this figure is available in the online journal.)

the observed X-ray sources as long as the relative CV to stellar density in the GB is comparable to the value in the local solar neighborhood.

Note there are a few caveats in this analysis. First, the radial distribution of the X-ray sources does not match well with the stellar distribution as shown in Figure 5(b). As mentioned, this is the reason for the large variation in the estimates of the relative CV density. It means the stellar model we use may not be appropriate for scaling the observed X-ray population directly. A solution could be found in some of the assumptions we have made. For instance, the (apparent) fraction of IPs in all CVs may not be constant across the fields.

Second, there are large uncertainties in the model parameters and various assumptions such as the ratio of IPs to all CVs and the fractional IPs with the X-ray luminosity $\geqslant 10^{32} \mathrm{erg} \mathrm{s}^{-1}$. For instance, according to Ritter \& Kolb (2003), the ratio of the known IPs to all known CVs are about $10 \%$, but this is also subject to a large uncertainty due to selection biases. Similarly, there is no firm estimate of the X-ray IP luminosity distribution to set the accurate limit for the fractional IPs with the X-ray luminosity $\geqslant 10^{32} \mathrm{erg} \mathrm{s}^{-1}$.

Third, as illustrated in the $\log N-\log S$ distributions of the $100 \mathrm{ks}$ and stacked data of the Sgr A* field, the X-ray variability can change the apparent source distribution. In order to understand the true distribution, it is necessary to monitor the GB fields continuously and extract the source distribution from a longer exposure. Considering the X-ray variability of the sources observed in the Sgr A* field, the true distribution of the GB X-ray population in the other GB fields can be $\gtrsim 20 \%-30 \%$ higher than what has been observed in the $100 \mathrm{ks}$ exposures.

A more recent study by Schödel et al. (2007) of the stellar population and mass content in the Galactic nucleus shows evidence for a higher mass contained in the central $\sim 1 \mathrm{pc}$ than predicted by studies and they speculate the excess may be due to a stellar remnant population such as black holes. Figure 6 shows the composition of the stellar components $\left(\rho_{A}\right.$, $\rho_{B}, \rho_{C}$, and $\left.\rho_{D}\right)$ in comparison with the observed projected $\mathrm{X}$-ray source density. Figure 6(a) shows the fit results using the stacked Sgr A* data (the same as Figure 5(b)) and Figure 6(b) for the $100 \mathrm{ks} \mathrm{Sgr} \mathrm{A*} \mathrm{data.} \mathrm{In} \mathrm{order} \mathrm{to} \mathrm{allow} \mathrm{the} \mathrm{possible} \mathrm{excess}$ of the X-ray population in the Galactic nucleus relative to the observable stellar population, we also fit the X-ray distribution by freeing the relative normalization parameter of the central spherical cluster component $\left(\rho_{A}\right)$ with respect to the rest of the components (Figures 6(c) and (d)). The resulting fits are substantially better, but they require a large excess of the central 
Table 5

Total X-ray and IR Surface Brightness (See Also Figure 7)

\begin{tabular}{|c|c|c|c|c|c|}
\hline \multirow[t]{2}{*}{ Field } & \multicolumn{2}{|c|}{$\begin{array}{c}\text { X-ray } \\
\text { a } \\
2-8 \mathrm{keV}\left(H_{C} \text { band }\right) \\
\left(10^{-10} \mathrm{erg} \mathrm{cm}^{-2} \mathrm{~s}^{-1} \mathrm{deg}^{-2}\right)\end{array}$} & \multicolumn{3}{|c|}{$\begin{array}{c}\mathrm{IR} \\
3.6 \mu \mathrm{m} \\
\left(\mathrm{MJy} \mathrm{sr}^{-1}\right) \\
\end{array}$} \\
\hline & PL & $\mathrm{PL}+\mathrm{Fe}$ & Observed & Unabsorbed (PL) ${ }^{\mathrm{b}}$ & Unabsorbed $(\mathrm{PL}+\mathrm{Fe})^{\mathrm{c}}$ \\
\hline BW & $0.34(0.02)$ & $0.35(0.02)$ & 7.4(0.4) & $8.2(0.4)$ & $8.2(0.4)$ \\
\hline SW & $0.48(0.01)$ & $0.49(0.01)$ & $15(1)$ & $17(2)$ & $17(2)$ \\
\hline LW & $1.10(0.01)$ & $1.13(0.01)$ & $18(1)$ & $24(1)$ & $24(1)$ \\
\hline Sgr B2 & $5.09(0.05)$ & $5.42(0.05)$ & $26(6)$ & $74(18)$ & $150(36)$ \\
\hline Sgr C & $14.9(0.1)$ & $16.6(0.1)$ & $35(3)$ & $152(13)$ & $384(33)$ \\
\hline Arches & $30.9(0.1)$ & $33.4(0.1)$ & $58(12)$ & 199(40) & $337(69)$ \\
\hline Sgr A* & $23.93(0.02)$ & $27.72(0.02)$ & $68(16)$ & $493(110)$ & $1100(250)$ \\
\hline
\end{tabular}

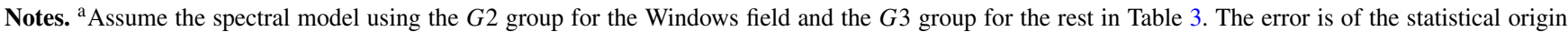
Note the expected CXB is $(1.6-1.7 \pm 0.2) \times 10^{-11} \mathrm{erg} \mathrm{cm}^{-2} \mathrm{~s}^{-1} \mathrm{deg}^{-2}$ for the Windows fields and $(1.3-1.4 \pm 0.2) \times 10^{-11} \mathrm{erg} \mathrm{cm}^{-2} \mathrm{~s}^{-1} \mathrm{deg}^{-2}$ for the rest (Hickox \& Markevitch 2006); see Section 5.2.

${ }^{\mathrm{b}}$ The $N_{\mathrm{H}}$ estimate by the PL (line B in Figure 7).

${ }^{c}$ The $N_{\mathrm{H}}$ estimate by PL+Fe (line A in Figure 7).

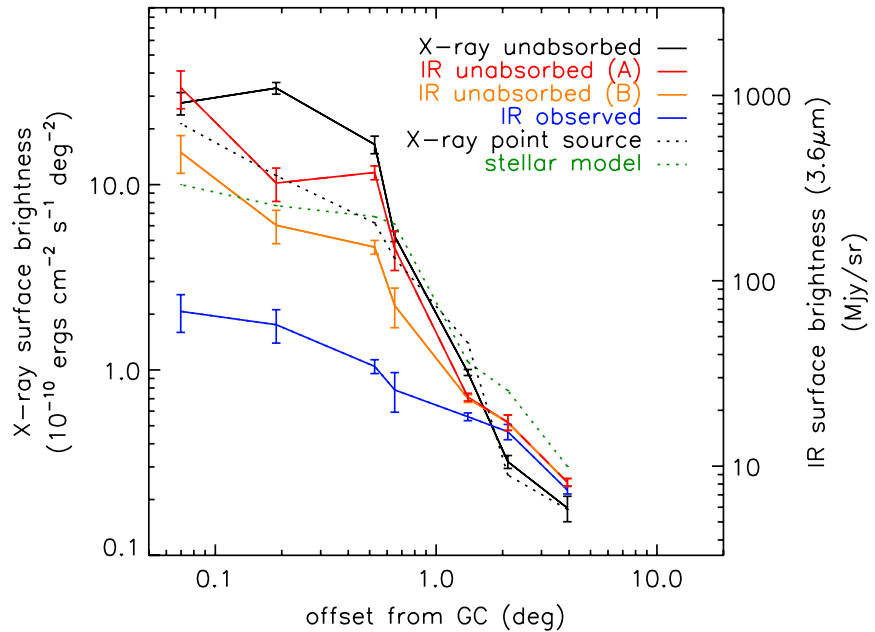

Figure 7. Comparison of the X-ray and the IR surface brightness. The X-ray surface brightness (black solid line) is the total unabsorbed $H_{C}$ flux minus the expected CXB flux in the Chandra ACIS-I FoV of each field assuming the quantile average spectral model (PL+Fe) of the $G 2$ (for the Windows fields) or $G 3$ group (for the rest). The infrared surface brightness (blue solid) is the average flux in the Chandra ACIS-I FoV from the $3.6 \mu \mathrm{m}$ band images by Spitzer/GLIMPSE. To calculate the unabsorbed IR flux consistently with the $\mathrm{X}$-ray flux, we assumed the $N_{\mathrm{H}}$ estimates by the quantile analysis in Table 3 ( $G 2$ for the Windows fields and $G 3$ for the rest). We use the $N_{\mathrm{H}}$ estimates by both models (A for PL and B for PL+Fe). We also assumed $A_{3.6 \mu \mathrm{m}}=0.5 A_{K s}$ (Nishiyama et al. 2009). The errors of the X-ray brightness are the quadratic sum of the statistical and systematic errors and the errors of the CXB estimates (see Section 5.2). The errors of the IR brightness are based on the statistical fluctuation of the brightness in the four ACIS-I fields, but the dominant errors in the high extinction fields come from the uncertainty in the extinction estimates as seen in the large difference of lines A and B. For easy comparison, we overlay the distributions of the X-ray source density (black dotted) and what the stellar model predicts (green dotted) without the AGN component using an arbitrary normalization factor.

(A color version of this figure is available in the online journal.)

stellar cluster component relative to the fixed normalization given in Section 4.3: $8.4 \times$ more for the stacked Sgr A* data and $4.5 \times$ more for the $100 \mathrm{ks}$ observation. This excess is still larger than the mass excess $(\sim 2 \times)$ of the nucleus above the observable stellar cluster estimated by Schödel et al. (2007), although the latter has a large uncertainty $(\sim 30 \%-50 \%)$.

\subsection{Total Surface Brightness}

Since the IR surface brightness is a good indicator of the stellar population, a direct comparison of the IR and X-ray surface brightness provides an independent clue on the origin of the X-ray emission in the fields without the influence of the uncertainties in the stellar model. In Table 5, we list the total X-ray and IR surface brightness of the seven fields. In Figure 7 , we compare the total IR surface brightness with the $\mathrm{X}$-ray surface brightness after removing the contribution from the cosmic X-ray background (CXB). The CXB is expected to be $(1.6-1.7 \pm 0.2) \times 10^{-11} \mathrm{erg} \mathrm{cm}^{-2} \mathrm{~s}^{-1} \mathrm{deg}^{-2}$ for the Windows fields and $(1.3-1.4 \pm 0.2) \times 10^{-11} \mathrm{erg} \mathrm{cm}^{-2} \mathrm{~s}^{-1} \mathrm{deg}^{-2}$ for the rest (Hickox \& Markevitch 2006). For easy comparison, Figure 7 also overlays the distributions of the X-ray source density and what the stellar model predicts without the AGN component in the dotted lines.

The X-ray surface brightness is calculated from the integrated counts in the $H_{C}$ band, using the quantile averaged model spectra of the $G 2$ group for the Windows fields and the $G 3$ group for the rest (Table 3 ). In order to subtract the instrumental background, we use the CXC background database constructed from stowed observations. For the relative normalization of the background subtraction, we use the integrated counts in the $10.5-12.5 \mathrm{keV}$. The error bars in Figure 7 are the quadratic sum of the statistical and systematic errors and the errors of the CXB estimates. For the statistical errors, we use the difference in the flux estimates between the PL and PL+Fe models.

The IR surface brightness is calculated from the $3.6 \mu \mathrm{m}$ mosaic images (1".2 pixel) generated by Galactic Legacy Infrared Mid-Plane Survey Extraordinaire (GLIMPSE) from the Spitzer/ IRAC observations (Churchwell et al. 2009). The error is based on the statistical variation of the surface brightness in the four Chandra ACIS-I FoVs of each field. In order to estimate the unabsorbed surface brightness consistently with the X-ray flux estimates, we assume the $N_{\mathrm{H}}$ estimates by the quantile analysis in Table 3 ( $G 2$ for the Windows fields and $G 3$ for the rest). We use the $N_{\mathrm{H}}$ estimates by both models (line A for PL and line B for PL $+\mathrm{Fe}$ in Figure 7) to explore the dependence of the $N_{\mathrm{H}}$ estimates. For a given $N_{\mathrm{H}}$ estimate, we use $N_{\mathrm{H}}=1.79 \times 10^{21} A_{V}$, $A_{K}=0.114 A_{V}=0.95 A_{K s}$, and $A_{3.6 \mu \mathrm{m}}=0.5 A_{K s}$ (Nishiyama et al. 2009). 
Table 6

Specific Luminosity of X-ray Point Sources

\begin{tabular}{|c|c|c|c|c|c|}
\hline Source & $\begin{array}{c}\text { Reported } \\
\left(10^{26} \mathrm{erg} \mathrm{s}^{-1} M_{\odot}^{-1}\right)\end{array}$ & $\begin{array}{l}\text { Energy } \\
\text { Range } \\
(\mathrm{keV})\end{array}$ & $\begin{array}{l}\text { Luminosity } \\
\text { Range } \\
\left(\mathrm{erg} \mathrm{s}^{-1}\right)\end{array}$ & $\begin{array}{c}\text { Scaled for } \\
10^{32.7-34} \mathrm{erg} \mathrm{s}^{-1} \text { in } 2-8 \mathrm{keV} \\
\left(10^{26} \mathrm{erg} \mathrm{s}^{-1} M_{\odot}^{-1}\right)\end{array}$ & Studied Fields \\
\hline This study & $0.5-2.8$ & $2-8$ & $10^{32.7-34}$ & $0.5-2.8$ & 7 GB fields (100 or $750 \mathrm{ks})$ \\
\hline M06 & $5.0 \pm 2$ & $0.5-8$ & $10^{32.7-34}$ & $3.3 \pm 1.3$ & $2^{\circ} \times 1^{\circ}$ around the GC $(100$ or $2 \times 12 \mathrm{ks})$ \\
\hline S06 & $45 \pm 9$ & $2-10$ & $10^{27-36}$ & $\begin{array}{l}9 \pm 3^{a} \\
6 \pm 2^{b}\end{array}$ & The local solar neighborhood \\
\hline R07 & $77 \pm 39$ & $2-10$ & $10^{30.3-32.3}$ & $3 \pm 1^{c}$ & The Sgr A* field (1 Ms) \\
\hline
\end{tabular}

Notes. M06: Muno et al. (2006), S06: Sazonov et al. (2006), R07: Revnivtsev et al. (2007).

${ }^{a}$ By the scaling by M06.

${ }^{\mathrm{b}}$ Figure 9 in S06.

${ }^{\mathrm{c}}$ Assuming $3 \%$ of the total emission $\left((4 \pm 2) \times 10^{27} \mathrm{erg} \mathrm{s}^{-1} M_{\odot}^{-1}\right)$ is from $>10^{32.7} \mathrm{erg} \mathrm{s}^{-1}$, based on Figure 6 in R07.

The radial distribution of the CXB-subtracted X-ray surface brightness roughly matches with the distribution of the unabsorbed IR surface brightness under the given uncertainties with the possible exception of the Arches field. This indirectly supports the idea that they share a common origin. In particular, the distribution of the unabsorbed IR surface brightness matches with the X-ray source distribution, showing higher concentration at the GC than the stellar distribution model. The origin of the discrepancy between the stellar distribution model and the IR surface brightness is unclear and requires further investigations, but note that the unabsorbed IR brightness in the high extinction fields depends sensitively on the extinction estimates, resulting in relatively large uncertainties. This dependence is also evident in the large variation among the ACIS-I FoVs in each field, which is at least in part due to the variation in the extinction across the FoVs. The radial distributions of the total $\mathrm{X}$-ray surface brightness (Figure 7) and the X-ray source density (Figure 5) also show a slightly different trend in a few fields such as the Arches field. For instance, the difference in the Arches field is due to the large contribution of a few bright sources in the total flux. We will address the detail of the unresolved $\mathrm{X}$-ray emission with respect to the identified point sources in a following paper (J. Hong et al. 2009, in preparation).

\subsection{Comparison with Other Results}

According to Equation (5) in M03, the X-ray source density in the $\mathrm{Sgr} \mathrm{A}^{*}$ field is $0.60 \pm 0.04 \mathrm{X}$-ray sources $\operatorname{arcmin}^{-2}$ at $S>1.5 \times 10^{-14} \mathrm{erg} \mathrm{cm}^{-2} \mathrm{~s}^{-1}$ or $1.25 \times 10^{-7} \mathrm{ph} \mathrm{cm}^{-2} \mathrm{~s}^{-1}$ in the 2-8 keV range under their assumption of a PL spectrum with $\Gamma=0.5$ and $N_{\mathrm{H}}=6 \times 10^{22} \mathrm{~cm}^{-2} .{ }^{12}$ This is roughly consistent with our results, $0.86 \pm 0.17 \mathrm{arcmin}^{-2}$ at $S>1.5 \times 10^{-14} \mathrm{erg}$ $\mathrm{cm}^{-2} \mathrm{~s}^{-1}$ from the stacked results under the PL+Fe model. The error is derived from the quadratic sum of the statistical error and $\sim 20 \%$ systematic errors (the difference between the PL and the PL+Fe model).

Table 6 summarizes a few estimates of the specific luminosity of the Galactic X-ray point sources in the Chandra/ACIS energy range in the literature. The range of the specific luminosity in our study is the variation among the seven fields under the assumption of the PL+Fe model for the X-ray spectra. Using Equation (7) in M06 and assuming the $\alpha$ values in Figure 5(a) and the number density of the X-ray sources $\left((0.3-1.8) \times 10^{-6}\right.$ X-ray sources $M_{\odot}^{-1}$ at $>1.1 \times 10^{32} \mathrm{erg} \mathrm{s}^{-1}$ ) in Table 4, we get

${ }^{12}$ M06 assume $\Gamma=1.5$ for the X-ray spectra of the sources in the $2^{\circ} \times 1^{\circ}$ region around the GC.
$(0.5-2.8) \times 10^{26} \mathrm{erg} \mathrm{s}^{-1} M_{\odot}^{-1}$ in $2-8 \mathrm{keV}$ for the luminosity range of $5 \times 10^{32}-10^{34} \mathrm{erg} \mathrm{s}^{-1}$.

M06 interpreted the result in Sazonov et al. (2006, hereafter S06) to be $1.0 \pm 0.3 \times 10^{27} \mathrm{erg} \mathrm{s}^{-1} M_{\odot}^{-1}$ for $10^{32.7-34} \mathrm{erg} \mathrm{s}^{-1}$ using Equation (5) in S06 and Equation (7) in M06, and claimed their result $\left(5 \pm 2 \times 10^{27} \mathrm{erg} \mathrm{s}^{-1} M_{\odot}^{-1}\right)$ is consistent with S06. However, the result in $\mathrm{S} 06$ is calculated in the $2-10 \mathrm{keV}$ range and M06 in $0.5-8 \mathrm{keV}$. In the $2-8 \mathrm{keV}$ range, the result in M06 becomes $3.3 \pm 1.3 \times 10^{26} \mathrm{erg} \mathrm{s}^{-1} M_{\odot}^{-1}$ under their assumption of $\Gamma=1.5$ and $N_{\mathrm{H}}=6 \times 10^{22} \mathrm{~cm}^{-2}$. Similarly, the result in S06 is scaled to be $9 \pm 3 \times 10^{26} \mathrm{erg} \mathrm{s}^{-1} M_{\odot}^{-1}$ in the same energy band. So there is a hint of mismatch in the results between M06 and S06 if one follows the scaling in M06, but also note that Figure 9 in S06 shows $\sim 6 \pm 2 \times 10^{26} \mathrm{erg} \mathrm{s}^{-1} M_{\odot}^{-1}$ in the $2-10 \mathrm{keV}$ band for $>10^{32-33} \mathrm{erg} \mathrm{s}^{-1}$, which is consistent with M06 and lower than the scaling done for S06 in M06. This conversion also reveals the result in M06 is consistent with our result for the Sgr A* field. Using a similar scaling based on Equation (5) in S06, we get $3 \pm 1 \times 10^{26} \mathrm{erg} \mathrm{s}^{-1} M_{\odot}^{-1}$ for the X-ray emissivity reported by Revnivtsev et al. (2007, hereafter R07) in the $2-8 \mathrm{keV}$ range for $10^{32.7-34} \mathrm{erg} \mathrm{s}^{-1}$, assuming $3 \%$ of the total emission in the same luminosity range based on Figure 6 in R07. Due to many different underlying assumptions in the above estimates (e.g., the spectral model parameters, stellar models, etc.), it is not easy to make a fair comparison among the reported results. The large uncertainties make these results appear consistent within $2 \sigma$, but our results are at the lower end of these findings.

M06 and Muno et al. (2009, hereafter M09) have presented the X-ray source distribution in a $2^{\circ} \times 1^{\circ}$ region around the GC. In the case of the $\log N-\log S$ distribution, one of the interesting results in M06 and M09 is a flatter distribution of the X-ray sources in the Arches Cluster and the subsequent excess of the X-ray sources near the high end of the flux range, compared to the Sgr A* field. We also see a similar cross over between the unstacked Sgr A* field and the Arches Cluster at $\sim 1.5 \times 10^{-13} \mathrm{erg} \mathrm{cm}^{-2} \mathrm{~s}^{-1}$ (or at $\sim 8 \times 10^{-13} \mathrm{erg} \mathrm{cm}^{-2} \mathrm{~s}^{-1}$ with the stacked Sgr A field, out of the range in Figure 5(a)). We believe this is a simple statistical fluctuation rather than a true representative of the population, arising from a small number of sources in the narrow FoV, where a few strong sources $(\sim 2-4$ in the Arches Cluster) skew the shape of the whole distribution. In fact, the small number statistics is also evident in the jumpy shape of the distributions near the high end of the flux range. In Figure 5 of M06, the excess of the X-ray sources in the Arches Cluster above $6 \times 10^{-6} \mathrm{ph} \mathrm{cm}^{-2} \mathrm{~s}^{-1}$ is boosted by excluding the overlapping region between the Sgr A* field and the Arches 
Cluster from the sky coverage calculation for the X-ray sources of the Arches Cluster. In order to minimize the effects due to the small number statistics, in our analysis, we focus on the solid line section of the distributions that contain at least 10 or more sources. In addition, quantile analysis results in a slightly higher value $(\sim 10 \%)$ of the rate-to-flux conversion factor for the $G 3$ sources in the Sgr A* field than the same for the Arches Cluster (see Figure 4), which in turn pushes the $\log N-\log S$ distribution of the Sgr A* field relatively higher. As a result, we find the slopes of the $\log N-\log S$ distributions of the Arches Cluster and Sgr A* fields are consistent and the Sgr A* field contains more X-ray sources than the Arches Cluster consistently below $\sim 3 \times 10^{-14} \mathrm{erg} \mathrm{cm}^{-2} \mathrm{~s}^{-1}$.

In the case of the radial distribution, the excess of the X-ray sources in the $\mathrm{Sgr} \mathrm{A}^{*}$ field with respect to the stellar model is about $3 \sigma$ above the stellar model if one considers both the statistical errors and the $\sim 20 \%$ systematic errors in the flux estimates (about $10 \sigma$ above only with the statistical errors). M09 found about $2.5 \sigma$ excess of the X-ray sources at the GC compared to the best-fit stellar model.

\subsection{Another Source Population in Sgr C (or Sgr B2)?}

The $1 / \theta$ distribution is roughly consistent with the observed radial distribution of the X-ray sources in the GB fields within $2 \sigma-3 \sigma$ except for the $\operatorname{Sgr} \mathrm{C}$ field. The $1 / \theta$ distribution is a merely empirical outcome if the observed X-ray source density consists of multiple components. On the other hand, the apparent $1 / \theta$ distribution along with the similar X-ray spectral properties may imply that the GB X-ray population belongs to a homogeneous component. If true, the excess of the $\mathrm{Sgr} \mathrm{C}$ and Sgr B2 fields can be simply viewed as the presence of another source population in these fields in addition to the population following the $1 / \theta$ distribution. The Sgr C field, like the Sgr B2 field, contains molecular $\mathrm{H}$ II complexes that host massive star formation. These molecular clouds are very luminous in hard $\mathrm{X}$-rays, in particular with the $6.4 \mathrm{keV}$ neutral iron line $(\mathrm{Mu}-$ rakami et al. 2001a, 2001b). In our analysis, the estimates of the $\Gamma$ value in the PL and PL+Fe models for the stacked spectra of the $G 3$ sources in the Sgr B2 and C fields are relatively lower compared to the rest of the fields, suggesting the possibility of another source population with a different spectral type that could be related to the star formation.

\section{CONCLUSION AND FUTURE WORK}

In the $\log N-\log S$ distribution of the sources in the GB fields, the systematic errors arising from certain assumptions of spectral type are usually disregarded due to lack of alternative approaches. However, they often dominate other systematic errors such as the EB, completeness or even statistical errors. The quantile analysis allows for a simple, robust method to assign a proper spectral type for flux calculation. The technique is shown to be reliable in the hard band $(>2 \mathrm{keV})$ and insensitive to the selection of the spectral model. In the soft band $(<2 \mathrm{keV})$, where the Galactic extinction has a great influence in the spectra, the result can vary drastically depending on the assumed spectral model class. Therefore, any results covering the soft energy range should be taken with caution.

The $\log N-\log S$ and radial distribution of the GB fields including the three low extinction Windows show the high concentration of the GB X-ray sources near the GC. The GB distribution clearly extends out to $\sim 1.4$ (LW) from the GC and possibly more. The spectral type of the GB X-ray sources appears to be largely consistent across the region under the PL model with an iron emission line at $6.7 \mathrm{keV}$. It is possible that one type of source constitutes the majority of the GB population, and the estimated X-ray density is consistent with the majority being magnetic CVs (IPs). The $1 / \theta$ relation of the radial distribution of the X-ray sources appears to be simply empirical in comparison with the stellar model compositions. Since the gravitational influence of the central supermassive black hole is only dominant within a few central pc (a few arcmin) at most, there is no compelling physics behind the $1 / \theta$ relation being fundamental out to a few degrees from the GC. However, the discrepancy between the stellar models and the X-ray distribution, and the apparent homogeneity of the $\mathrm{X}$-ray spectral properties of these X-ray sources pose other possibilities: the $1 / \theta$ relation may not be so empirical or at least the X-ray source population contains some components that are not easily traceable by the visible stellar population.

The radial distributions of the total X-ray and IR surface brightness in the fields match within the given uncertainties, implying the same origin. The radial distribution of the IR surface brightness resembles the distribution of the X-ray point sources perhaps better than that predicted by stellar distribution models, but a further detailed analysis is required because of the sensitive dependence of the IR surface brightness on the extinction estimates in the high extinction fields. In the case of the X-ray surface brightness, the additional care must be taken due to dominant contributions of a few bright sources on the X-ray flux in star formation fields such as the Arches field.

While multi-wavelength observational campaigns provide important clues on the GB X-ray population, the true nature of GB X-ray sources may not be completely resolved due to source confusion and high obscuration. A deep observation ( $\sim 1 \mathrm{Ms})$ of the LW (Revnivtsev et al. 2009), designed to investigate the nature of the Galactic Ridge X-ray emission (GRXE) in the field, is very encouraging for studies of the nature of X-ray point sources in the GB. Such a deep observation allows a direct detection of iron emission lines or X-ray variability in many of the GB X-ray sources, with which we can identify the nature of individual sources. We note only a handful of sources in the $1 \mathrm{Ms}$ data of the $\operatorname{Sgr} \mathrm{A}^{*}$ field had enough statistics for identification through such a direct discovery (M03; M04). But the low extinction in the Window fields can be a game changer. For instance, we have identified an IP in the BW from the $100 \mathrm{ks}$ observation, based on the periodic X-ray modulation associated with the X-ray spectral change (Hong et al. 2009). According to its average flux, the source can be a bright IP $\left(\sim 10^{33} \mathrm{erg} \mathrm{s}^{-1}\right)$ near the GC, but for a similar source in the Sgr $A^{*}$ field it would be very difficult to observe such a spectral change or periodic modulation due to the heavy absorption. By a crude scaling based on one IP found in the $100 \mathrm{ks}$ observation of the BW, one can expect about 30-40 such identifications in a $1 \mathrm{Ms}$ exposure of the BW. ${ }^{13}$ Such findings would also provide enough statistics to explore the radial distribution of this particular source type. Therefore, continuous X-ray monitoring of the low extinction Window fields including the SW and $\mathrm{BW}$ is another important approach for unveiling the nature of the GB X-ray sources. Note that the Window fields are also suitable for searching non-magnetic CVs in the GB. These are

\footnotetext{
13 Assume the identifiable source distribution is proportional to $S_{\mathrm{th}}{ }^{-3 / 2}$, where $S_{\text {th }}$ gets 10 times fainter, and also assume an additional $20 \%-30 \%$ increase in the probability of catching highly variable X-ray sources based on the difference in the stacked and unstacked data set of the Sgr A* field.
} 
potentially more abundant than magnetic CVs, but they are known to have relatively soft spectra and thus they would be likely hidden in the high extinction fields such as the Sgr A* field.

This work is supported in part by NASA/Chandra grants GO6-7088X, GO7-8090X and GO8-9093X. We thank the referee for the insightful comments and discussions.

\section{REFERENCES}

Ak, T., et al. 2008, New Astron., 13, 133 Baganoff, F. K., et al. 2003, ApJ, 591, 891

Bogdanov, S., Grindlay, J. E., \& van den Berg, M. 2005, ApJ, 630, 1029

Cappelluti, N., et al. 2005, A\&A, 430, 39

Churchwell, E., et al. 2009, PASP, 121, 213

Ebisawa, K., et al. 2005, ApJ, 635, 214

Freeman, P. E., et al. 2002, ApJS, 138, 185

Gehrels, N., et al. 1986, ApJ, 303, 336

Grindlay, J. E., et al. 2005, ApJ, 635, 907

Heinke, C. O., et al. 2008, in AIP Conf. Proc. 1010, A Population Explosion, ed. R. M. Bandyopadhyay et al. (Melville, NY: AIP), 136

Hickox, R. C., \& Markevitch, M. 2006, ApJ, 645, 95

Hong, J., Schlegel, E. M., \& Grindlay, J. E. 2004, ApJ, 614, 508 (H04)

Hong, J., et al. 2005, ApJ, 635, 907 (H05)

Hong, J., et al. 2009, ApJ, 699, 1053
Kim, D.-W., et al. 2004, ApJS, 150, 19

Kim, E., et al. 2007, ApJ, 659, 29

Kent, S. M., Dame, T. M., \& Fazio, G. 1991, ApJ, 378, 131

Launhardt, R., Zylka, R., \& Mezger, P. G. 2002, A\&A, 384, 112

Laycock, S., et al. 2005, ApJ, 634, L53 (L05)

Muno, M. P., et al. 2003, ApJ, 589, 225 (M03)

Muno, M. P., et al. 2004, ApJ, 613, 1179 (M04)

Muno, M. P., et al. 2005, ApJ, 633, 228

Muno, M. P., et al. 2006, ApJS, 165, 173 (M06)

Muno, M. P., et al. 2009, ApJS, 181, 110 (M09)

Murakami, H., et al. 2001a, ApJ, 550, 297

Murakami, H., et al. 2001b, ApJ, 558, 687

Nishiyama, S., et al. 2009, ApJ, 696, 1407

Picaud, S., \& Robin, A. C. 2004, A\&A, 428, 891

Pretorius, M. L., et al. 2007, MNRAS, 382, 1279

Revnivtsev, M., Vikhlinin, A., \& Sazonov, S. 2007, A\&A, 473, 857 (R07)

Revnivtsev, M., et al. 2009, Nature, 458, 1142

Ritter, H., \& Kolb, U. 2003, A\&A, 404, 301 (update RKcat7.10)

Robin, A. C., et al. 2003, A\&A, 409, 523

Ruiter, A., Belczynski, K., \& Harrison, T. 2006, ApJ, 640, L167 (R06)

Sazonov, S., et al. 2006, A\&A, 450, 117 (S06)

Schlegel, D., Finkbeiner, D., \& Davis, M. 1998, ApJ, 500, 525

Schödel, R., et al. 2007, A\&A, 469, 125

Stanek, K. Z. 1998, arXiv:astro-ph/9802307

van den Berg, M., Hong, J., \& Grindlay, J. 2009, ApJ, 700, 1702

Wang, Q. D., Dong, H., \& Lang, C. 2006, MNRAS, 371, 38 (W06)

Wang, Q. D., Gotthelf, E. V., \& Lang, C. C. 2002, Nature, 415, 148

Wijnands, R., et al. 2005, ApJ, 618, 883 